\title{
Nonpersistence of Breather Families for the Perturbed Sine Gordon Equation
}

\author{
Jochen Denzler \\ Mathematisches Institut, Ludwig-Maximilians-Universität, Theresienstr. 39, D-80333 München, \\ Germany \\ e-mail: 〈jochen.denzler@mathematik.uni-muenchen.dbp.de〉
}

Received: 7 December 1992/in revised form: 26 April 1993

\begin{abstract}
We show that, up to one exception and as a consequence of first order perturbation theory only, it is impossible that a large portion of the well-known family of breather solutions to the sine Gordon equation could persist under any nontrivial perturbation of the form

$$
u_{t t}-u_{x x}+\sin u=\varepsilon \Delta(u)+O\left(\varepsilon^{2}\right),
$$

where $\Delta$ is an analytic function in an arbitrarily small neighbourhood of $u=0$. Improving known results, we analyze and overcome the particular difficulties that arise when one allows the domain of analyticity of $\Delta$ to be small. The single exception is a one-dimensional linear space of perturbation functions under which the full family of breathers does persist up to first order in $\varepsilon$.
\end{abstract}

\section{Introduction}

\subsection{The Problem}

Nontrivial solutions to a wave equation are called breathers if they decay as $|x| \rightarrow \infty$ ( $x$ the space variable) and are periodic in time $t$. A wave equation known to admit such solutions is the sine Gordon equation

$$
u_{t t}-u_{x x}+\sin u=0 \text {. }
$$

As it can be viewed as a completely integrable Hamiltonian system, explicit solutions are known, in particular the family of breathers

$$
u^{*}(x, t)=u^{*}(x, t ; m)=4 \arctan \frac{m}{\omega} \frac{\sin \omega t}{\cosh m x}, \quad m, \omega>0, m^{2}+\omega^{2}=1 .
$$

We are concerned with the question whether this type of solution exists for other nonlinear Klein Gordon equations

$$
u_{t t}-u_{x x}+g(u)=0, \quad\left(g(0)=0, g^{\prime}(0) \neq 0\right)
$$


nearby, i.e. where the nonlinearity $g(u)$ is close to $\sin u$. There are reasons (explained in Sect. 1.3) for suspecting that existence of breathers is a very rare and singular phenomenon, and in fact our result roughly says that the family of breathers does not persist under any nontrivial perturbation of the specified kind. The next section gives the precise statement of this result, and discusses its limitations.

A nonexistence result for breathers has already been explained by Segur and Kruskal in [23], but no proof in full mathematical rigor is given there. Our nonpersistence result is based on a similar result by Birnir, McKean, and Weinstein [5], and we begin by following closely their approach. However, additional analysis permits us to get the same result under considerably weaker hypotheses. Our result has been presented on a conference on dynamical systems in Sankt Petersburg, and an announcement is to appear [10].

There are trivial perturbations of the sine Gordon equation which arise merely by a scaling of $(x, t)$ and $u$ in the unperturbed equation. A non-persistence result has to cope with the fact that breathers do persist under scalings.

We now formulate the theorem in all details.

\subsection{The Result}

Theorem 1. Consider the perturbed sine Gordon equation

$$
u_{t t}-u_{x x}+\sin u=\varepsilon \Delta(u, \varepsilon)=\varepsilon \Delta(u)+O\left(\varepsilon^{2}\right) .
$$

Let $\Delta(u, 0)=: \Delta(u)$ and $\Delta(0, \varepsilon)=0$ for all $\varepsilon$. Suppose that $\Delta(\cdot, \cdot)$ is $C^{1}$ in both variables and analytic in the first variable in a neighbourhood of $(0,0)$, say

$$
\left\{(u, \varepsilon)|| \tan \frac{u}{4}|<\varrho \leq 1,| \varepsilon \mid<\varepsilon_{0}\right\} .
$$

(i) Then it is possible to rescale $(x, t)$ and $u$ in such a way that in the rescaled variables (called $x, t, u$ again), $\varepsilon \Delta(u, \varepsilon)$ does not contain terms of order $u^{1}$ or $u^{3}$ (but possibly such of order $\left.u^{2}\right)$. The scaling normalizes only the odd part of the function $\Delta(\cdot, \varepsilon)$, but does not change the even part.

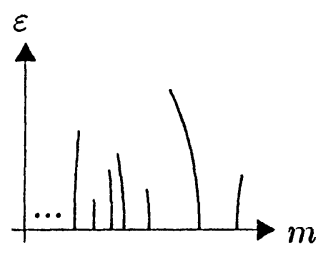

(ii) Assume in addition this scaling to be performed, i.e. that $\varepsilon \Delta(u, \varepsilon)$ does not contain $u^{1}$ or $u^{3}$ terms.

Suppose that for infinitely many values of $m$ satisfying $0<\frac{m}{\omega} \leq \varrho^{\prime}<\varrho$ there exists a breather solution $u_{m}(x, t, \varepsilon)$ of time period $T_{m}(\varepsilon)=\frac{2 \pi}{\omega_{m}(\varepsilon)}$ to $(1.4)_{\varepsilon}$ for $0<\varepsilon<\varepsilon_{\max }(m)$ which depends $C^{1}$ on $\varepsilon$ as a function from $\left[0, \varepsilon_{\max }(m)[\right.$ to $\mathbf{X}$, such that the periods $T_{m}(\varepsilon)$ depend continuously on $\varepsilon$, and such that $u_{m}(x, t, 0)=u^{*}(x, t ; m)$. 
Here, $\mathbf{X}$ is the normal space of time-periodic and spatially decaying functions that is defined in the beginning of Sect.2.1. Then, the first order of the perturbation can (up to a constant factor) only be the even function

$$
\Delta(u)=\frac{1}{4}\left(1-4 \cos \frac{u}{2}+3 \cos u+4 \cos u \ln \cos \frac{u}{4}\right)=-\frac{9}{32} u^{2}+O\left(u^{4}\right) .
$$

(iii) If there persist infinitely many breathers as before and if $\Delta(\cdot, \cdot)$ and $u_{m}(\cdot, \cdot, \cdot)$ are also analytic with respect to $\varepsilon$ and if moreover $\Delta(u, \varepsilon)$ does not contain a quadratic term in $u$, then $\varepsilon \Delta(u, \varepsilon)$ is a scaling of the sine Gordon equation.

In fact, we can show nonperistence for the exceptional perturbation (1.5), too [11]. This result, however, needs second order perturbation theory. Its proof is deferred to a forthcoming paper.

Several remarks help to explore the merits and the limitations of the theorem:

(1) Our result contains the parameter $0<\varrho \leq 1$, which determines the domain in which analyticity of the perturbation function needs to be assumed. At the same time, it determines the amplitudes $m$ for which persistence of the breather $u^{*}(\cdot, \cdot ; m)$ is a well-defined notion: the range of $u_{m}(\cdot, \cdot, \varepsilon)$ is close to the range of $u^{*}(\cdot, \cdot ; m)$ and must be contained in the domain of $\Delta(\cdot, \varepsilon)$. The result by Birnir, McKean, and Weinstein mentioned before, concerns the case $\varrho=1$. On the other hand, Theorem 1 deals with nonlinearities that are analytic only in an arbitrarily small neighbourhood of 0 , i.e. $\varrho$ can be any small positive number. This seemingly innocent improvement is not a mere technicality, but requires new ideas. Together with the elimination of (1.5) given in [11], it is our basic improvement compared to Birnir, McKean, and Weinstein [5]. It should however be clear that this further progress was possible only on the base laid by these authors.

(2) We need not assume persistence in the sense that $u(x, t, \varepsilon)$ exists for all $\varepsilon \in$ $\left[0, \varepsilon_{\max }[\right.$; a sequence $\varepsilon \rightarrow 0$ is enough.

(3) We have not assumed $\varepsilon_{\max }(m)$ to be uniform in $m$. Neither is any uniformity in $m$ for $\left\|u_{m}\right\|_{\mathbf{x}}$ needed.

(4) The theorem implies in particular that except for scalings and the specified perturbation (1.5) that is left over, there exists some $m_{\max }$ such that no breather with $m<m_{\max }$ persists.

(5) The proof of Theorem 1 relies entirely on the first order equations in $\varepsilon$. In other words, simultaneous persistence of infinitely many breathers can be excluded by linearization arguments alone.

Theorem 1 can be complemented by further results: For instance, persistence of a single breather cannot be excluded by linearization arguments. More precisely, we can show [11] that for any fixed $0<\frac{m}{\omega}<1$, there exists an infinite dimensional linear space of first order perturbation functions $\Delta(\cdot)$ for which the sine Gordon breather persists up to first order in $\varepsilon$. We are not able to decide persistence of a single breather by means of higher orders of the perturbation, nor do we know whether first order perturbation theory is sufficient to rule out simultaneous persistence of some finite number of breathers.

We have not touched at all the global (in $\varepsilon$ ) problem, i.e. the general nonlinear Klein Gordon equation. However, the proof of Theorem 1 will lead to a few illuminating, though speculative, glimpses on this equation (see Sect. 4.2).

We have stressed our point that Theorem 1 can deal with arbitrarily small domains of analyticity of the perturbation, i.e. with arbitrarily small $\varrho$. However, the assumption $\varrho \leq 1$ is artificial, and there is the opportunity for an improvement in the other 
direction, too. One may wish to exclude the persistence of infinitely many large amplitude breathers $\left(\frac{m}{\omega}>1\right)$ for any nontrivial entire perturbation function. Theorem 1 cannot handle this case because of the condition $\varrho \leq 1$. This kind of restriction will be removed in Sect. 4.1, where we prove the following theorem as a corollary to Theorem 10 .

Theorem 2. Suppose the analyticity requirement for $\Delta(\cdot, \cdot)$ in the beginning of Theorem 1 is relaxed such that $\Delta$ need be analytic in the first variable only in some complex neighbourhood of the real segment $-\varrho^{\prime} \leq \tan \frac{u}{4} \leq \varrho^{\prime}<\infty$. Then, if infinitely many breathers persist in the interval $0<\frac{m}{\omega} \leq \varrho^{\prime}$, the conclusions of Theorem 1 hold without change.

The technical point is that discussions in the neighbourhood of $\frac{m}{\omega}=0$ involve a series expansion argument that does not reach beyond $\left|\frac{m}{\omega}\right|<1$ even for entire $\Delta(\cdot)$. For Theorem 2, one has to replace the series by an integral representation, which is however not appropriate near $\frac{m}{\omega}=0$. Both together produce the full information.

\subsection{Background}

Physicists consider breathers as particle-like solutions of some (classical) field theory. Non-existence of breathers for $u_{t t}-u_{x x}+u-u^{3}=0$ has been claimed and discussed in a physical context by Segur and Kruskal [23], but not proved rigorously. There is a large number of other contexts in which nonlinear Klein Gordon equations arise in physics. Some of them can be found in [3], where further references are given. Let us mention a heuristic common sense argument against the existence of breathers. Asking for a breather solution means posing a boundary value problem to a wave equation. Such problems are typically ill-posed.

One can view the nonlinear Klein Gordon equation (1.3) as a dynamical system

$$
\partial_{x} U=\left[\begin{array}{cc}
0 & 1 \\
\partial_{t}^{2}+g(\cdot) & 0
\end{array}\right] U
$$

on some space of periodic functions (of the variable $t$ ). Then, the dynamical variable is $x$. Here,

$$
U(x)=\left[\begin{array}{c}
u(x, \cdot) \\
u_{x}(x, \cdot)
\end{array}\right]
$$

The linearization of this equation about its trivial solution $U \equiv 0$ has the matrix $A \equiv\left[\begin{array}{cc}0 & 1 \\ \partial_{1}^{2}+g^{\prime}(0) & 0\end{array}\right]$. Considered as an operator on $L^{2}\left(\mathbb{R} / T \mathbb{Z}, \mathbb{R}^{2}\right), A$ has pure point spectrum

$$
\operatorname{spec} A=\left\{ \pm \sqrt{g^{\prime}(0)-\left(\frac{2 n \pi}{T}\right)^{2}} \mid n \in \mathbb{Z}\right\}
$$




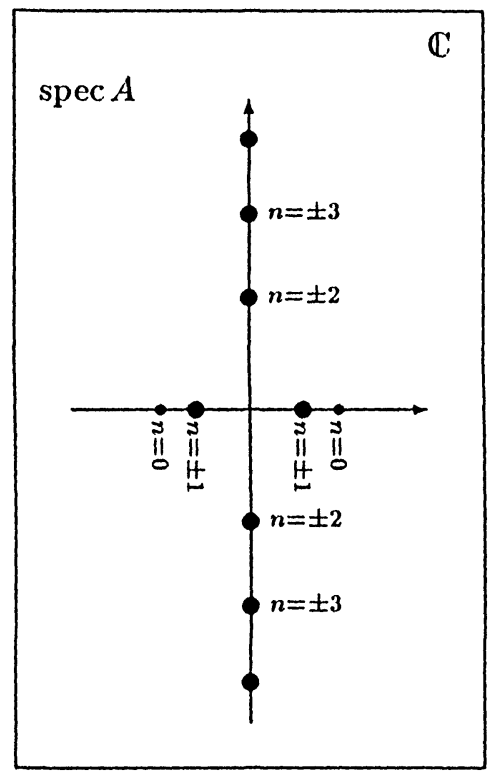

In this setting, a breather solution is a homoclinic orbit to the fixed point $U=0$. This point of view has been stressed by [27,12]. If a breather exists, this means that miraculously the finite dimensional stable and unstable manifolds coincide or at least have a one dimensional orbit in common in an infinite dimensional space. These manifolds may split under perturbation of the equation. A non-persistence result means that they do actually split. We shall consider some obstructions whose vanishing is a necessary condition for the persistence of breathers under the perturbation. These obstructions should therefore be related somehow to some geometrical object like splitting distance or splitting angle. No such connection is established by now. But one fact is remarkable: The obstructions to be considered will turn out to be exponentially small with respect to the amplitude of the unperturbed breather. This situation reminds one of the results on exponentially small splitting of separatrices established recently $[9,14,18,22]$. Probably the analogy is not too close, because the problems with the exponentially small terms (which are notoriously difficult) turn out to be comparatively easy in our setting.

If the stable and unstable manifold picture is more than only heuristics, it means in particular that for $g^{\prime}(0)<(2 \pi / T)^{2}$, these manifolds are one dimensional, so any $T$-periodic solution that decays either as $x \rightarrow \infty$ or $x \rightarrow-\infty$ corresponds to the $n=0$ Fourier component, i.e. is independent of $t$. Exactly this has been proved by Coron [6] in 1982. His proof does not even mention invariant manifolds, but is quite an elementary calculation. However, the idea of invariant manifolds is still in the background behind his calculation. Recently, Kichenassamy [16, Theorem 5] proved a stable manifold theorem directly for this situation.

Let us now consider the same equation, but with $t$ as the dynamical variable, as a dynamical system on some space of decaying functions (of the variable $x$ ). Then a breather is simply a periodic solution. We want to compare the situation with Lyapunov's center theorem. It says: Let $\dot{u}=A u+O\left(u^{2}\right)$ be an ordinary differential equation having an integral of motion, and let $\pm i \lambda$ be a pair of simple imaginary eigenvalues of $A$. (It gives therefore rise to a 2 dimensional linear space $\Sigma$ fibred by 
periodic solutions to the linear equation $\dot{u}=A u$.) Now, if $\pm i n \lambda(n=2,3, \ldots)$ are not contained in the spectrum of $A$, then locally a 2 dimensional manifold $S$ fibred by periodic solutions to the nonlinear equation exists, and it is tangential to $\Sigma$ in 0 . Without the last nonresonance condition, $S$ might not exist.

Even if we have no PDE version of Lyapunov's center theorem at hand, let us look at the linearization about 0 and see whether the nonresonance conditions are satisfied.

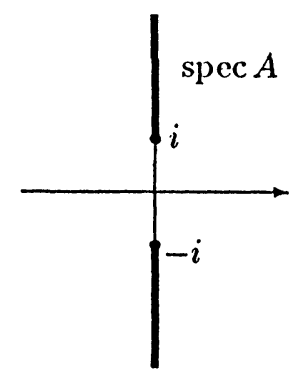

This is what (1.1) now looks like:

$$
\partial_{t} U=\left[\begin{array}{cc}
0 & 1 \\
\partial_{x}^{2}-\sin (\cdot) & 0
\end{array}\right] U, \quad U=\left[\begin{array}{c}
u \\
u_{t}
\end{array}\right]
$$

The linearization about 0 has the matrix $A=\left[\begin{array}{cc}0 & 1 \\ \partial_{x}^{2}-1 & 0\end{array}\right]$ with continuous spectrum all on the imaginary axis: $\operatorname{spec} A= \pm i \cdot[1, \infty[$. We have no eigenvalues (and no periodic solution that could correspond to a pair of eigenvalues), only continuous spectrum. This naive model does therefore not display an analogy with Lyapunov's center theorem.

The reason is that the breather (1.2) is a genuinely nonlinear phenomenon, not visible in the linearization about $0 . u^{*}$ spreads out on the real axis as $m \rightarrow 0$, and the shape ceases to decay for $|x| \rightarrow \infty$ in the limit $m \rightarrow 0$. This degeneracy near $u=0$ is the culprit for the exponential smallness of the obstructions mentioned above. When proving Theorem 1 for a perturbation function $\Delta$ that is analytic only in some arbitrarily small neighbourhood of 0 , one is forced to work near this degeneracy. This is one way to explain, why our theorem needs new ingredients beyond the theorem by Birnir, McKean, and Weinstein.

Nevertheless, there is an analogy with Lyapunov's center theorem, which we are going to pursue now. It will justify that the continuous spectrum in the above picture is indeed responsible for resonances with the unperturbed breather, and these resonances are obstructions to the persistence of the breather. The right way to talk about spectra for a nonlinear equation like sine Gordon is to talk about the spectral parameter in the (linear) scattering problem associated to it: The sine Gordon equation (like other soliton equations) can be studied by associating with it a linear equation that contains an eigenvalue parameter $\lambda$. In this linear equation (called the associated scattering problem), the sine Gordon wave $u\left(\cdot, t_{0}\right)$ for fixed $t_{0}$ plays the role of a potential, and $\lambda$ plays the role of the energy of wave functions $\psi$ that are scattered by this potential. The important connection between the two problems is that the scattering data (a term that can be given a precise meaning, but we need not do so for the purposes of this exposition) do not depend on $t_{0}$, provided $u(\cdot, \cdot)$ solves the sine Gordon equation, even though the scattering problem does depend on $t_{0}$ through the potential $u\left(\cdot, t_{0}\right)$. 
In other words, time evolution of the nonlinear wave $u(\cdot, t)$ according to the sine Gordon equation describes an isospectral deformation of the potential $u(\cdot, t)$ in the associated scattering problem with deformation parameter $t$.

Resuming our comparison with Lyapunov's center theorem, let us describe the resonances that occur and are responsible for the nonpersistence. For first order perturbation theory, we shall have to consider the linearized operator

$$
\mathscr{L}:=\partial_{t}^{2}-\partial_{x}^{2}+\cos u^{*}
$$

about the sine Gordon breather $u^{*}$, and the functions in the kernel of $\mathscr{L}$ are asymptotic to free Klein Gordon waves $e^{i k x+i \Omega t}$ (where $k^{2}+1=\Omega^{2}$ ). The continuous spectral parameter $\lambda$ from the associated scattering problem determines the wave number $k=k(\lambda)$ and the frequency $\Omega=\Omega(\lambda)$ of these free Klein Gordon waves. It is a good idea to associate the above picture of the continuous spectrum with the possible values of $i \Omega(\lambda)$. The breather potential also admits bound states of the scattering problem, which correspond to certain complex values of $\lambda$, and the associated frequencies are $\pm \omega$. Now, $\pm i \omega$ plays the role that would be played by the simple pair of eigenvalues in Lyapunov's center theorem. There is a resonant interaction of the breather with the continuous spectrum similar to the resonances that have to be excluded in Lyapunov's center theorem, when integer multiples $\pm i n \omega$ of $\pm i \omega$ fall into the continuous spectrum $\{i \Omega(\lambda) \mid \lambda \in \mathbb{R}\}= \pm i[1, \infty[$. In fact, we have such a resonance for every integer $n \neq 0, \pm 1$, provided $\omega>\frac{1}{2}$, i.e. $m<\sqrt{3} / 2$. This gives infinitely many necessary conditions (one for each $n$ ) for the persistence.

Generically, one cannot expect these conditions to be satisfied. Genericity results have been obtained by Sigal [25]. He includes potential terms and allows any space dimension, and discusses the problem in general spectral theoretic terms. Our results are more detailed, but hold only for a very special situation.

The responsibility of these conditions for the nonpersistence result can also be illustrated as follows: When one modifies the problem and looks for time periodic solutions to the perturbed sine Gordon equation on a finite $x$-interval, say with Dirichlet boundary conditions, then the continuous spectrum is replaced by a point spectrum. In this case, there is a fair chance to avoid the resonances, but it is very delicate: with infinitely many eigenvalues $\pm i \Omega_{k}$, one has to expect "near miss" encounters where $\left|n \omega-\Omega_{k}\right|$ becomes arbitrarily small. So one gets persistence results for a Cantor set of periods, but one has to cope with small denominator problems; see $[7,8,17]$.

\section{Proof of the Theorem}

\subsection{First Order Perturbation Theory and Outline of Proof}

In order to be definite about the periodicity and decay assumptions, let

$$
\mathbf{X}=\left\{u(\cdot, \cdot) \in C^{2}(\mathbb{R} \times \mathbb{R} / 2 \pi \mathbb{Z})|| u(x, t)|+| u_{x}(x, t)|+| u_{t}(x, t) \mid \leq \frac{c}{1+x^{2}}\right\} .
$$

The smallest possible $c$ can be taken as a norm, making $\mathbf{X}$ a (dense) subspace of a Banach space (of weighted $C^{1}$-functions). The particular choice of the function space is not really important for the following arguments, however. We say sloppily 
that a $T$-periodic (in the second argument) function $u$ is in $\mathbf{X}$, if the function $(x, t) \mapsto u\left(x, t \frac{T}{2 \pi}\right)$ is in $\mathbf{X}$.

The basic argument by which one begins is contained in the next lemma; it is a necessary condition for the persistence of a breather:

Lemma 3 (First order perturbation theory). Consider the perturbed sine Gordon equation $(1.4)_{\varepsilon}$. Suppose that $(u, \varepsilon) \mapsto \varepsilon \Delta(u, \varepsilon)$ is $C^{1}$ with respect to both variables in a neighbourhood of $(0,0)$, say in $\left\{(u, \varepsilon)|| \tan \frac{u}{4}|<\varrho \leq 1,| \varepsilon \mid<\varepsilon_{0}\right\}$. Also consider the family of breathers (1.2) that solve the unperturbed sine Gordon equation $(1.4)_{0}$. Suppose that one (for $m=m_{0}$, such that $m_{0} / \sqrt{1-m_{0}^{2}}<\varrho$ ) of these breathers persists under the perturbation, in other words, that for $0 \leq \varepsilon<\varepsilon_{\max }$, there exists a $T(\varepsilon)$-periodic (in $t$ ) classical solution $u(x, t, \varepsilon)$ to $(1.4)_{\varepsilon}$ satisfying $u(x, t, 0)=u^{*}\left(x, t ; m_{0}\right)$, such that $\varepsilon \mapsto u(\cdot, \cdot, \varepsilon),\left[0, \varepsilon_{\max }\left[\rightarrow \mathbf{X}\right.\right.$ is a $C^{1}$-function and $T(\cdot)$ is continuous.

Then $v(x, t):=\left.\partial_{\varepsilon}\right|_{\varepsilon=0}\left(u(x, t, \varepsilon)-u^{*}(x, t ; m(\varepsilon))\right)$ solves the equation

$$
\mathscr{L} v:=\left(\partial_{t}^{2}-\partial_{x}^{2}+\cos u^{*}\right) v=\Delta\left(u^{*}\right),
$$

where $u^{*}$ denotes the function $u^{*}\left(\cdot, \cdot ; m_{0}\right)$.

Proof. We can write

$$
u(x, t, \varepsilon)=u^{*}(x, t ; m(\varepsilon))+\varepsilon v(x, \tau)+o(\varepsilon),
$$

where

$$
\tau=t T(0) / T(\varepsilon) \text { and } m(\varepsilon)=\sqrt{1-(2 \pi / T(\varepsilon))^{2}},
$$

and $v$ is $T(0)$-periodic in the second variable. Inserting (2.2a) in (1.4) $)_{\varepsilon}$ gives

$$
\underbrace{u_{t t}^{*}-u_{x x}^{*}+\sin u^{*}}_{=0}+\varepsilon \mathscr{L} v+o(\varepsilon)=\varepsilon \Delta\left(u^{*}+O(\varepsilon), \varepsilon\right) .
$$

Taking the leading order in $\varepsilon$ gives the claimed result.,

Remark. Note that $t$, not $\tau$ has entered in $u^{*}$. This has the effect that the $0^{\text {th }}$ order approximation $u^{*}(x, t, m(\varepsilon))$ is an exact solution to the unperturbed equation, but that $m$ varies with $\varepsilon$. If one chose to adapt the period by writing $u^{*}(x, \tau, m)$ instead of $u^{*}(x, t, m(\varepsilon)$ ), one would get an equivalent ansatz (with a different $v$ ) all right, but one that messes up the different orders of the perturbation.

A necessary condition for the breather at $m$ to persist is that the equation $\mathscr{L} v=\Delta\left(u^{*}\right)$ can be solved for $v$, i.e. that $\Delta\left(u^{*}\right) \in \operatorname{im} \mathscr{L}^{\text {formally }}(\operatorname{ker} \mathscr{L})^{\perp}$. Now, a sequence of functions $\chi_{n} \in \operatorname{ker} \mathscr{L}$ can be given, so we have infinitely many conditions for each $m$, namely $\mathscr{B}_{n}:=\int \chi_{n} \Delta\left(u^{*}\right) d x d t=0$. We have also infinitely many degrees of freedom: the perturbation function $\Delta(\cdot)$.

It turns out that $\mathscr{B}_{n}$ is a function of $\frac{m}{\omega}$ that is exponentially small in $\frac{m}{\omega}$. On the other hand, to prove the theorem means to show that $\mathscr{R}_{n}$ does not vanish in a neighbourhood of $\frac{m}{\omega}=0$. This difficulty is resolved by the fact that an exponentially small factor that is given explicitly can be split off from $\mathscr{B}_{n}$, leaving a term $\tilde{\mathscr{B}}_{n}$ 
that is an analytic function of $\frac{m}{\omega}$ near 0 . This is done in Sect. 2.3 and is borrowed from [5].

A further reduction kills the scalings, for which persistence is known a priori. In this step, the role of the exceptional perturbation (1.5) becomes clearer: it sneaks in like the shadow of one of the scalings. As the even and the odd parts of the perturbation are treated separately (but in full analogy) in all of the proof, the roles played by the scalings affect only the odd part, whereas (1.5) lies in the even part and plays the very same role there. A side effect of this further reduction is a radical simplification of the formulas, without which the key to the result for arbitrarily small analyticity domain could hardly have been discovered.

This key is one more surprising explicit formula. We argue that nothing short of this formula could have provided the qualitative information that is needed to conclude the proof, because extracting this information from the available data is ill-conditioned, which reflects the degeneracy of the problem near $\frac{m}{\omega}=0$ again.

We now complete the proof by filling in the details.

\subsection{Normalizations} Suppose that $\varepsilon \Delta(u, \varepsilon)=\sum_{1}^{\infty} a_{j}(\varepsilon) u^{j}$. Transforming $(1.4)_{\varepsilon}$ by the scalings $(\check{x}, \check{t})=$
$(\mu x, \mu t), \breve{u}=\lambda u$, we get $=$

$$
\check{u}_{\check{t} \check{t}}-\check{u}_{\check{x} \check{x}}+\sin \check{u}=\varepsilon \Delta\left(\frac{\check{u}}{\lambda}, \varepsilon\right)+\sin \check{u}-\frac{\lambda}{\mu^{2}} \sin \frac{\check{u}}{\lambda} .
$$

Therefore, we see that in order to get rid of the $\check{u}^{1}$ - and $\check{u}^{3}$-terms, we need $a_{1}=1-\mu^{2}$ and $a_{3}=-\left(1-\mu^{2} \lambda^{2}\right) / 6$. Here, $a_{1}$ and $a_{3}$ are of order $\varepsilon$, and the equations can be solved for $\lambda, \mu=1+O(\varepsilon)$. The claim on the parity is also obvious from this formula. So we have proved (i), and we may assume from now on that the normalization by scalings has already been performed. However, this normalization will be exploited only at the end of the proof.

Let us note in passing that the scaling with $\lambda(\varepsilon)=1+\lambda^{\prime} \varepsilon+\ldots$ and $\mu(\varepsilon)=$ $1+\mu^{\prime} \varepsilon+\ldots$ adds $\left(2 \mu^{\prime}-\lambda^{\prime}\right) \sin u+\lambda^{\prime} u \cos u$ to the first order $\Delta(u)$ of the perturbation function.

Next take care of the translation invariance (in $x$ and $t$ ) of $(1.4)_{\varepsilon}:$ If the perturbed equation has breathers, then their translates in time and space are also breather solutions. The unperturbed breather (1.2) can be distinguished from its translates in time and space by the normalization condition $u^{*}(0,0 ; m)=u_{x}^{*}\left(0, \frac{\pi}{2 \omega} ; m\right)=0$. By means of the implicit function theorem one can impose the analogous condition on $u_{m}$, thus breaking the translation invariance. When we write

$$
u_{m}(x, t, \varepsilon)=u^{*}(x, t ; m(\varepsilon))+\varepsilon v_{m}(x, \tau)+o(\varepsilon),
$$

according to Lemma 3 , this normalization has the consequence that

$$
v(0,0)=v_{x}\left(0, \frac{\pi}{2 \omega}\right)=0
$$

with analogous equations for all higher order terms (in $\varepsilon$ ) $v^{[2]}, \ldots$, if we can expand further. 
In the formulas that follow, we write atn for the arc tangent and ch, sh, th for the hyperbolic functions.

\subsection{Calculating the Obstructions}

We have seen in the outline of the proof that necessary conditions for persistence of the breather at $\frac{m}{\omega}$ is the solvability of the equation $\mathscr{L} v=\Delta\left(u^{*}\right)$. We exhibit appropriate test functions for this equation:

Lemma 4. For every integer $n$ and either sign of $\sqrt{n^{2} \omega^{2}-1}=: k_{n}$,

$$
\begin{aligned}
\chi_{n, k_{n}}(x, t)= & e^{i k_{n} x+i n \omega t}\left(1+\frac{m^{2} \sin ^{2} \omega t}{\omega^{2} \operatorname{ch}^{2} m x}\right)^{-1} \\
& \times\left\{\frac{k_{n}}{2 m} \operatorname{th} m x+\frac{n}{2} \frac{\cos \omega t \sin \omega t}{\operatorname{ch}^{2} m x}\right. \\
& \left.-i\left[\left(\frac{k_{n}}{2 m}\right)^{2}-\frac{1}{4}\right]-i \frac{n^{2}+1}{4} \frac{\sin ^{2} \omega t}{\operatorname{ch}^{2} m x}\right\}
\end{aligned}
$$

is a $2 \pi / \omega$-periodic solution to the linearized sine Gordon equation $\mathscr{L} \chi=0$. Provided $\omega>\frac{1}{2}$, the $\chi_{n}$ are bounded for $|n| \geq 2$. On the other hand, $\chi_{0}$ grows exponentially as $x \rightarrow \pm \infty$. The solutions for $n= \pm 1$,

$$
\chi_{1, i m}(x, t)=\frac{1}{2}\left(1+\frac{m^{2} \sin ^{2} \omega t}{\omega^{2} \operatorname{ch}^{2} m x}\right)^{-1} \times\left\{\frac{i e^{\imath \omega t}}{\operatorname{ch} m x}+\frac{\sin \omega t e^{-m x}}{\operatorname{ch}^{2} m x}\right\}
$$

and $\chi_{1,-i m}(x, t)=\chi_{1, \imath m}(-x, t), \chi_{-1, \pm i m}(x, t)=\chi_{1, \pm i m}(x,-t)$, decay as $x \rightarrow \pm \infty$ and correspond to the time and space derivatives of the sine Gordon breather:

$$
\begin{gathered}
\partial_{t} u^{*}=-4 i m\left(\chi_{1, i m}+\chi_{-1, i m}\right)=-4 i m\left(\chi_{1,-i m}+\chi_{-1,-i m}\right), \\
\partial_{x} u^{*}=4 m^{2} / \omega\left(\chi_{1, i m}-\chi_{1,-i m}\right)=4 m^{2} / \omega\left(\chi_{-1,-i m}-\chi_{-1, i m}\right) .
\end{gathered}
$$

Proof. These functions $\chi_{n, k_{n}}(x, t)$ are special cases of the solutions $\Phi_{2}^{A}(x, t, \lambda) e^{\imath \Omega(\lambda) t}$ to $\mathscr{L} \chi=0$ exhibited in the appendix. $\lambda$ is chosen such that $\Omega(\lambda)=n \omega$, i.e. that $\chi_{n, k_{n}}$ have the time periodicity of the breather. The equation $\Omega(\lambda)=n \omega$ clearly displays the resonance of the breather with the continuous spectrum.

The calculations of $\partial_{t} u^{*}$ and $\partial_{x} u^{*}$ are immediate.

Remark 1. For $x \rightarrow \pm \infty, \chi_{n, k_{n}}$ is asymptotic to a constant times $e^{\imath k_{n} x+\imath n \omega t}$, a free wave solution to the Klein Gordon equation. This is no surprise, because under this limit, $u^{*} \rightarrow 0$, so $\mathscr{L}$ tends to the Klein Gordon operator $\partial_{t}^{2}-\partial_{x}^{2}+1$. All the other factors in the formula for $\chi_{n, k_{n}}$ are "Nature's camouflage" in the sense that all qualitative features in the formulas that will follow and their physical interpretation depend only on the exponential term. In particular, the relation $k_{n}^{2}=(n \omega)^{2}-1$ is the dispersion relation for Klein Gordon waves.

Multiplying $\mathscr{L} v=\Delta\left(u^{*}\right)$ with $\chi_{n, k_{n}}$ and integrating by parts yields

$$
\mathscr{R}_{n}^{\Delta}\left(\frac{m}{\omega}\right):=\int_{-\infty}^{\infty} \int_{0}^{2 \pi / \omega} \chi_{n, \pm k_{n}}(x, t) \Delta\left(u^{*}(x, t)\right) d t d x=0 .
$$


Note that $\mathscr{R}_{n}^{\Delta}$ is independent of the sign in $k_{n}$, because $\chi_{n,-k_{n}}(x, t)=\chi_{n, k_{n}}(-x, t)$ and $u^{*}$ is even in $x$. We shall simplify notation by writing $\chi_{n}$ for $\chi_{n, k_{n}}$ and $\mathscr{R}_{n}$ for $\mathscr{R}_{n}^{\Delta}$, when no confusion can arise.

At present, $0<\frac{m}{\omega}<1$, and we consider $z=\frac{m}{\omega}$ as the independent variable. Then, $m=z / \sqrt{1+z^{2}}$, and $\omega=1 / \sqrt{1+z^{2}}$. Later, we shall consider $z$ as a complex variable.

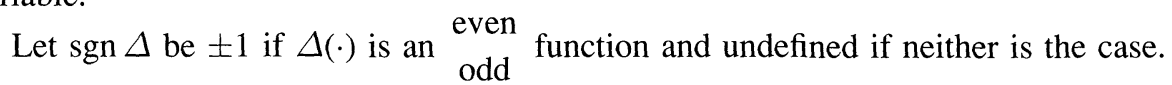
Obviously, $\mathscr{R}_{n}$ is linear in $\Delta$, so up to a linear decomposition we may assume $\Delta$ is either even or odd. A glance at the $t$-harmonics involved shows that $\mathscr{R}_{n}$ vanishes if $n$ and $\Delta$ have opposite parity. Moreover,

$$
\mathscr{R}_{-n}^{\Delta}\left(\frac{m}{\omega}\right)=(\operatorname{sgn} \Delta) \mathscr{R}_{n}^{\Delta}\left(\frac{m}{\omega}\right), \quad \mathscr{R}_{n}^{\Delta}\left(-\frac{m}{\omega}\right)=\mathscr{R}_{n}^{\Delta}\left(\frac{m}{\omega}\right) .
$$

$\mathscr{R}_{ \pm 1}^{\Delta}$ vanishes identically for all $\Delta$ : For even ones due to the $t$-integration, for odd ones due to the $x$-integration, because the odd (in $t$ ) part of $\chi_{1, \pm i m}$ is also odd in $x$.

The definition of the $\mathscr{B}_{n}$ leads us to the basic splitting lemma

Lemma 5. Let $\Delta(\cdot)$ be analytic about 0 ; define the sequence $\left(\Delta_{p}\right)$ by the Taylor expansion

$$
\Theta(z):=\Delta(4 \operatorname{atn} z) /\left(1+z^{2}\right)=\sum_{p=1}^{\infty} \Delta_{p} z^{p}, \quad|z|<\varrho \leq 1
$$

and formally let $\Delta_{-1}:=\Delta_{0}:=0$. Then we can decompose

$$
\mathscr{R}_{n}^{\Delta}\left(\frac{m}{\omega}\right)=\left(i \frac{m}{\omega}\right)^{n-1} \frac{\pi^{2}}{2 m^{2} \omega^{2}} E_{n}\left(\frac{m}{\omega}\right) \tilde{\mathscr{R}}_{n}^{\Delta}\left(\frac{m}{\omega}\right) \quad \text { for } 0<\frac{m}{\omega}<\varrho \leq 1,
$$

where $E_{n}$ does not vanish, and $\tilde{\mathscr{R}}_{n}(z)$ can be continued to an analytic function for $|z|<\varrho$. In detail,

$$
E_{n}\left(\frac{m}{\omega}\right)=\prod_{r=-\left[\frac{n-1}{2}\right]}^{-1}\left[r(n+r)+\frac{n^{2}-1}{4 m^{2}}\right] \begin{cases}\frac{k_{n} / 2 m}{\operatorname{sh}\left(\pi k_{n} / 2 m\right)} & \text { for } n \geq 2 \text { even } \\ \frac{1}{\operatorname{ch}\left(\pi k_{n} / 2 m\right)} & \text { for } n \geq 3 \text { odd }\end{cases}
$$

and

$$
\begin{aligned}
\tilde{\mathscr{R}}_{n}^{\Delta}\left(\frac{m}{\omega}\right)= & \sum_{q=0}^{\infty}\left(\frac{m^{2}}{\omega^{2}}\right)^{q}\left(\begin{array}{c}
n+2 q \\
q
\end{array}\right)[(n+2 q-1) !]^{-1} \prod_{r=0}^{q-1}\left[r(n+r)+\frac{n^{2}-1}{4 m^{2}}\right] \\
& \times\left\{\Delta_{n+2 q}\left(\frac{n^{2}+1}{2} \omega^{2}-1-\frac{n^{2} \omega^{2}-1}{n+2 q}\right)\right. \\
& \left.+\Delta_{n+2 q-2}\left(\frac{n^{2}+1}{2} \omega^{2}+\frac{n^{2} \omega^{2}}{n+2 q}\right)\right\} .
\end{aligned}
$$



Consequently, the equation $\mathscr{L} v=\Delta\left(u^{*}\right)$ has a solution $v$, only if ${ }^{1} \tilde{\mathscr{B}}_{n}^{\Delta}\left(\frac{m}{\omega}\right)=0$
for all integers $n \geq 2$.

Proof of the Lemma. For the straightforward calculation of $\mathscr{R}_{n}$, one has to insert the series for $\Theta$ and the formula for $\chi_{n}$ (Lemma 4) into the integral (2.3). Depending on whether $n$ is even or odd, only the even or odd component of $\Delta$ enters. So, one can write $p=n+2 q$ for the summation index (or $p=n+2 q-2$ for two terms, for which one shifts the index). In the course of the calculation, the following integrals appear:

$$
\begin{gathered}
\int_{0}^{2 \pi / \omega} \sin ^{n+2 q} \omega t e^{i n \omega t} d t=\frac{2 \pi}{\omega}(-1)^{n+q} \frac{1}{(2 i)^{n+2 q}}\left(\begin{array}{c}
n+2 q \\
q
\end{array}\right), \quad(n>0), \\
\int_{-\infty}^{\infty} \frac{e^{i k x}}{\operatorname{ch}^{p} m x} d x=\frac{1}{m} \begin{cases}\frac{2^{p-1}}{(p-1) !} \frac{\pi}{\operatorname{ch} \frac{\pi k}{2 m}} \prod_{\tilde{j}=1 / 2}^{p / 2-1}\left(\tilde{j}^{2}+\frac{k^{2}}{4 m^{2}}\right) & \text { for } p \text { odd, } m>0 \\
\frac{2^{p-1}}{(p-1) !} \frac{\pi k / 2 m}{\operatorname{sh} \frac{\pi k}{2 m}} \prod_{\tilde{j}=1}^{p / 2-1}\left(\tilde{j}^{2}+\frac{k^{2}}{4 m^{2}}\right) & \text { for } p \text { even, } m>0\end{cases}
\end{gathered}
$$

(The terms containing th $m x$ or $\cos \omega t$ can be reduced to the above integrals, too, by integration by parts.) The detailed calculation has been done in [5].

With the explicit formula at hand, we can cancel $E_{n} \neq 0$ from $\mathscr{B}_{n}^{\Delta}\left(\frac{m}{\omega}\right)=0$ now and are left with the persistence condition $\tilde{\mathscr{B}}_{n}^{\Delta}\left(\frac{m}{\omega}\right)=0$ as claimed.

The calculation automatically yields that the series (2.6) converges for $\left|\frac{m}{\omega}\right|<\varrho$ (after the obvious cancellation of $m^{2 q}$ has been executed in the first line). Therefore, $\tilde{\mathscr{B}}_{n}\left(\frac{m}{\omega}\right)$ is analytic in this disc. Since the theorem assumes persistence of infinitely many breathers, the corresponding values of $\frac{m}{\omega}$ must accumulate somewhere; it must be in the interior of the domain of analyticity of $\tilde{\mathscr{B}}_{n}^{\Delta}$, because we have explicitly ruled out the boundary by the condition $\frac{m}{\omega} \leq \varrho^{\prime}<\varrho$. Therefore, we can conclude, following [5], that $\tilde{\mathscr{R}}_{n}^{\Delta}$ vanishes identically, and we may exploit this by inserting complex values of $\frac{m}{\omega}$.

All the rest of the proof relies on this analytic continuation argument. The condition that all $\tilde{\mathscr{B}}_{n}^{\Delta}$ vanish identically in a neighbourhood of 0 will determine a four dimensional space of coefficient sequences $\left(\Delta_{p}\right)$. These four dimensions correspond to the two scalings, the perturbation (1.5) not accounted for geometrically, and the shift of $u$ that is ruled out by the condition $\Delta(0)=0$.

In particular, if $\frac{m}{\omega}$ takes certain purely imaginary values, determined by the zeros of the product in $(2.6)$, then $\tilde{\mathscr{B}}_{n}^{\Delta}\left(\frac{m}{\omega}\right)$ reduces to a finite sum, and the condition

1 The condition is also sufficient according to the completeness result described in the appendix: see Theorem 12 
$\tilde{\mathscr{R}}_{n}^{\Delta}\left(\frac{m}{\omega}\right)=0$ becomes a recursion for the coefficients $\Delta_{p}$ : For any positive integer $\tilde{q}$, select $\frac{m}{\omega}$ according to

$$
\begin{aligned}
\frac{n^{2}-1}{4 m^{2}}= & -\tilde{q}(\tilde{q}+n) \Leftrightarrow m^{2}=\frac{1-n^{2}}{(n+2 \tilde{q})^{2}-n^{2}} \\
& \Leftrightarrow \frac{m^{2}}{\omega^{2}}=\frac{1-n^{2}}{(n+2 \tilde{q})^{2}-1}=: z^{2}(n, \tilde{q})
\end{aligned}
$$

for integers $n \geq 2$ and $\tilde{q} \geq 1$. Then the only non-vanishing terms of the sum (2.6) are those with $\bar{q}=0,1, \ldots, \tilde{q}$, and hence the sum involves $\Delta_{n-2}, \Delta_{n}, \ldots, \Delta_{n+2 \tilde{q}}$, altogether $\tilde{q}+2$ coefficients. We let

$$
R^{\Delta}(n, \tilde{q}):=\frac{(-1)^{[n / 2]}}{2 \omega^{2}\left(1-n^{2}\right)} \tilde{\mathscr{R}}_{n}^{\Delta}(z(n, \tilde{q})), \quad \text { if }|z(n, \tilde{q})|<\varrho,
$$

and this does not depend on which one of the two values of $\frac{m}{\omega}$ defined by (2.7) we denote as $z(n, \tilde{q})$ and which one as $-z(n, \tilde{q})$. We postpone writing out formulas for $R(n, \tilde{q})$ until after the further reduction of $\tilde{\mathscr{R}}_{n}$ in the following section. Then the strange normalization factor just introduced will cancel.

Remark 2. As promised earlier, $\tilde{\mathscr{R}}_{n}$ is exponentially small with respect to $\frac{m}{\omega}$ as $\frac{m}{\omega} \rightarrow 0$. This happens, because in (2.3), an exponential factor $e^{i k_{n} x}$ is integrated against an analytic function $\Delta\left(u^{*}\right)$ that decays like $1 / \operatorname{ch} m x$. On the natural length scale $1 / m$ for $x$, the exponential is highly oscillatory.

Remark 3. The resonances of the basic frequency $\omega$ of the breather with frequencies of the continuous spectrum of Klein Gordon waves lead to poles of $\mathscr{B}_{n}$ (or $E_{n}$ ) on the imaginary axis in the complex $\frac{m}{\omega}$-plane. These poles are exactly at the points $z(n, \tilde{q})$ defined by (2.7), as can be seen easily. They accumulate at 0 for every fixed $n$. So, if $R^{\Delta}(n, \tilde{q})=0$ for infinitely many $\tilde{q}$, then $\tilde{\mathscr{B}}_{n}^{\Delta} \equiv 0$ by analytic continuation.

\subsection{Further Reducing the Obstructions}

The coefficients $\Delta_{p}$ introduced in (2.4) may be viewed as coordinates on the space of perturbations.

We already know that there must be a two dimensional space of perturbations, generated by the scaling solutions

$$
\begin{gathered}
\Delta(u)=\sin u \Leftrightarrow \Delta_{2 n+1}=4(-1)^{n}(n+1)^{2} \\
\Delta(u)=u \cos u \Leftrightarrow \Delta_{2 n+1}=4(-1)^{n} \sum_{k=0}^{n} \frac{(2(n-k)+1)^{2}}{2 k+1},
\end{gathered}
$$

for which all $\tilde{\mathscr{B}}_{n}$ vanish identically, because breathers trivially persist under scalings. (One can insert these sequences into the recursions as a sensitive check against calculational errors.) 
This makes it desirable to have coordinates on the quotient space of all perturbation functions modulo the scalings. The coordinates $\mathrm{A}_{p}$ described below come close to being such coordinates. Introducing them divides out the scalings, but also the additional perturbation (1.5). The latter will therefore be recovered in the very end, when the perturbations are reconstructed from the $\mathrm{A}_{p}$-coordinates.

We postpone a more detailed discussion and the necessary proofs into Sect. 3 and simply state the result here:

Lemma 6. Given a sequence $\left(\Delta_{p}\right)_{p \geq 0}$ that does not grow faster than coefficients of a convergent power series can, define its sequence of weighted second differences, $\left(A_{p}\right)_{p \geq 2}$ by

$$
\Gamma_{p}:=(-1)^{\lfloor p / 2\rfloor} \frac{\Delta_{p}}{(p+1)^{2}}, \quad \mathrm{~B}_{p}:=p\left(\Gamma_{p}-\Gamma_{p-2}\right), \quad \mathrm{A}_{p}:=\mathrm{B}_{p+2}-\mathrm{B}_{p} .
$$

Then, $\tilde{\mathscr{R}}_{n}^{\Delta}\left(\frac{m}{\omega}\right)$, given by (2.6), can be expressed in terms of $\mathrm{A}_{p}$ as follows:

$$
\tilde{\mathscr{R}}_{n}^{\Delta}\left(\frac{m}{\omega}\right)=C \sum_{q=0}^{\infty} \frac{\mathrm{A}_{n+2 q}}{q !(n+q) !}\left(\frac{-1}{\omega^{2}}\right)^{q} \prod_{r=0}^{q}\left[\frac{n^{2}-1}{4}+r(n+r) m^{2}\right]
$$

with $C=2(-1)^{\lfloor n / 2\rfloor} \omega^{2}\left(1-n^{2}\right)$.

Remark 4. We do not write down the general formula for $R(n, \tilde{q})$ in the $\Delta_{p}$ coordinates. There is no more need to do so, once the $\mathrm{A}_{p}$-coordinates are available. However, the formula $R(n, 1)=0$ played an important role in discovering (2.9). It reads

$$
n \frac{\Delta_{n-2}}{(n-1)^{2}}+2(n+1) \frac{\Delta_{n}}{(n+1)^{2}}+(n+2) \frac{\Delta_{n+2}}{(n+3)^{2}}=0 .
$$

The reduction formulas were constructed in such a way as to make the conditions $R(n, 1)=0$ trivial. Then it is quite natural to try how other obstructions simplify in the new coordinates. This project leads to Lemma 6.

The proof of this lemma consists of two partial summations; it will be given in detail in Sect. 3.1. We intend to argue that $\tilde{\mathscr{R}}_{n}^{\Delta} \equiv 0$ for all $n$ implies $\mathrm{A}_{p}=0$ for all $p$. Then, going back from $\mathrm{A}_{p}$ to $\Delta_{p}$ will select the scaling solutions for odd $p$ and (1.5) for even $p$. No further solution is selected for even $p$ because of the condition $\Delta(0)=0$, i.e. $\Delta_{0}=0$. This will then complete the proof of part (ii) of the theorem.

Let us explicitly write down the recursions $R(n, \tilde{q})$. Inserting (2.7) into (2.6), we find that

$$
R^{\mathrm{A}}(n, \tilde{q})=\sum_{q=0}^{\tilde{q}-1} \frac{\mathrm{A}_{n+2 q}}{q !(n+q) !}\left(-\frac{n^{2}-1}{(n+2 \tilde{q})^{2}-1}\right)^{q} \prod_{r=0}^{q}[\tilde{q}(n+\tilde{q})-r(n+r)] .
$$

(We feel free to specify either $\Delta$ or $\mathrm{A}$ as a superscript to $R$, when the perturbation function should be expressed, or to specify nothing. This should not lead to confusion.)

In order to determine $\mathrm{A}_{p}$, we can use all those conditions $R(n, \tilde{q})=0$ for which $|z(n, \tilde{q})|<\varrho$, because the conclusion $\tilde{\mathscr{R}}_{n}^{\Delta} \equiv 0$ is valid only in a disc of radius $\varrho$. For $\varrho=1$, this is no restriction: $|z(n, \tilde{q})|<1$ for all integers $n \geq 2$ and $\tilde{q} \geq 1$. In this case,

$$
0=R(n, 1)=(n+1) \mathrm{A}_{n} / n !
$$




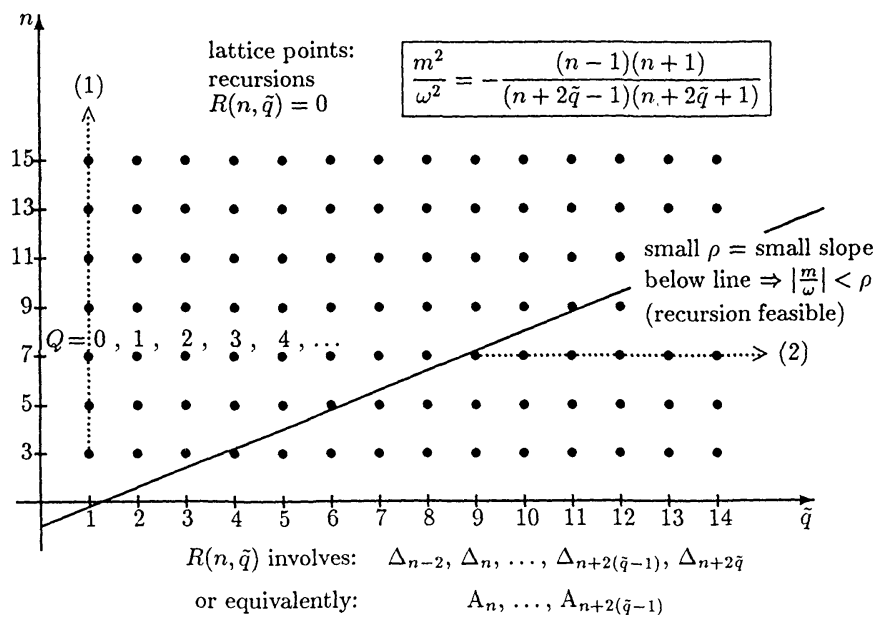

Fig. 1. The finite recursions.

(1) Recursive calculation of coefficients selects scaling solutions (feasible only for $\varrho=1$ ), (2) recursive calculation of coefficients selects solutions to $\tilde{\mathscr{B}}_{n} \equiv 0$ for $\left|\frac{m}{\omega}\right|<\varrho$ (any $\varrho$; ill-
conditioned; no problem due to surprising explicit formula). The equation of the separating straight line is $\frac{n}{n+2 \tilde{q}}=\varrho^{1 / 2}$. The condition that $(n, \tilde{q})$ lies below
it is slightly stronger than $|z(n, \tilde{q})|<\varrho$

immediately proves the desired result. This is the situation considered by Birnir, McKean, and Weinstein [5]. The argument works just as easily in the $\Delta$-coordinates. $\Delta_{1}$ and $\Delta_{3}$ are initial conditions (giving the scalings), and then $R(n, \tilde{q})=0$ determines $\Delta_{3+2 \tilde{q}}$ recursively for $\tilde{q}=1,2,3, \ldots$.

For $\varrho<1$, there are severe restrictions, because $z(n, \tilde{q}) \rightarrow \pm i$ for $\tilde{q}$ fixed and $n \rightarrow \infty$, so the only way to get information on all power series coefficients $\mathrm{A}_{n+2 \tilde{q}}$ is to let $\tilde{q} \rightarrow \infty$, i.e. to work with conditions involving more and more terms. See Fig. 1 for the situation (in case $n$ is odd).

Remark5. Since $\mathrm{A}_{n}=n ! R(n, 1) /(n+1)$, one can read (2.11) in the following way: All conditions $R(n, \tilde{q})=0$ are consequences of the conditions $R(n, 1)=0$, the shortest recursions. Translated back into the $\Delta_{p}$-coordinates, this means that all higher $(\tilde{q}+2)$-term-recursions are consequences (linear combinations) of the 3-termrecursions. Therefore, the latter contain already all information that is contained in $\tilde{\mathscr{B}}_{n} \equiv 0$.

Remark6. One can attribute a geometric (physical) meaning to the different conditions. To fix $n$ means to fix a test function $\chi_{n} \in \operatorname{ker} \mathscr{L}$, in other words, to look at the $n^{\text {th }}$ Fourier component in time. We shall do this immediately.

To fix $\frac{m}{\omega}$, considering $\tilde{\mathscr{B}}_{n}\left(\frac{m}{\omega}\right)$ for varying $n$ (no recursion, but an infinite series), means to study first order persistence of one specific breather.

But to fix $\tilde{q}$ means to look at a different breather for every Fourier component, and to consider the first resonance belonging to each Fourier component.

The condition $R(n, \tilde{q})=0$ is a vanishing pole condition: It is equivalent to the condition that the possible pole of $\tilde{\mathscr{B}}_{n}(z)$ at $z=z(n, \tilde{q})$ does not occur. 


\subsection{Explicit Solution for the Recursion}

For every $n$, we have conditions $R(n, \tilde{q})=0$ to determine $\left(\mathrm{A}_{p}\right)$, where $\tilde{q} \geq \tilde{q}_{\min }(n, \varrho)$, and $\tilde{q}_{\min }$ is large if $\varrho$ is small. So, we have a $\left(\tilde{q}_{\min }-1\right)$-dimensional space of solutions determined by arbitrary initial conditions $\mathrm{A}_{n}, \ldots, \mathrm{A}_{2 \tilde{q}_{\mathrm{m} \text { In }}-4}$ and the available recursions $R(n, \tilde{q})=0$.

We want to show that the intersection of these finite dimensional spaces for all different $n$ is $\{0\}$. In this procedure, the key step is that an explicit formula for this solution space can be found. All we need from this formula will be the asymptotic behaviour of a solution sequence $\left(\mathrm{A}_{p}\right)$. These asymptotic expansions will turn out to be linearly independent for a collection of different $n$, leaving only the zero solution in the intersection. We give the lemmas here and postpone their proofs until Sect. 3.

$$
R(n, \tilde{q})=\sum_{q=0}^{\tilde{q}-1} \frac{\mathrm{A}_{n+2 q}}{q !(n+q) !}\left(-\frac{n^{2}-1}{(n+2 \tilde{q})^{2}-1}\right)^{q} \prod_{r=0}^{q}[\tilde{q}(n+\tilde{q})-r(n+r)]
$$

Lemma 7. Let $n$ be fixed and consider the recursions $R(n, \tilde{q})=0$ for variable $\tilde{q}$. For any nonnegative integer $Q$, the following sequence $\mathrm{A}_{n+2 q}^{(Q)}$ is the "Greens function" at $Q$ for these recursions, i.e. $R^{\mathrm{A}^{(Q)}}(n, \tilde{q})=0$ for $\tilde{q} \neq Q+1$, but is $\neq 0$ for $\tilde{q}=Q+1$,

$$
\begin{gathered}
\mathrm{A}_{n+2 q}^{(Q)}=T(n, q, Q) \alpha^{2 q}, \quad \alpha^{2}=\frac{(n+2 Q+1)(n+2 Q+3)}{n^{2}-1}, \\
T(n, q, Q)=\frac{q(q-1) \ldots(q-Q+1) \cdot(n+2 q+1)(n+2 q+3)}{(q+n+1)(q+n+2) \ldots(q+n+Q+2)} .
\end{gathered}
$$

For $Q=0$, the term $q(q-1) \ldots(q-Q+1)$ is to be interpreted as the empty product, 1.

In particular, for $n$ fixed, it follows $\mathrm{A}_{n+2 q}^{(Q)} \sim\left(4 \alpha^{-n}\right) \alpha^{n+2 q}$ as $q \rightarrow \infty$.

For $n$ fixed and $0 \leq Q \leq \tilde{q}_{\min }(n, \varrho)-2$, the $\alpha=\alpha(n, Q)$ are all different, therefore any solution $\left(\mathrm{A}_{p}\right)$ to $\bar{R}(n, \tilde{q})=0, \tilde{q} \geq \tilde{q}_{\min }$ is asymptotic to $c \alpha^{p}$ for some $c$ and some $\alpha$ among the finitely many values determined by the lemma. This argument holds for all $n$. But according to the following lemma, no real number $\alpha^{2}$ is of the form given in Lemma 7 for every $n$ at once. So the constant $c$ must vanish, hence $\mathrm{A}_{p}=0$.

Lemma 8. Suppose that a number $\alpha>0$ has the property that either for every large enough even $n$ or for every large enough odd $n$, there exists $N=N(n)$ such that $\alpha$ is of the form $\alpha=\frac{N^{2}-1}{n^{2}-1}$. Then $N=n$ and $\alpha=1$.

We have just proved that $\mathrm{A}_{p}=0$ for all $p \geq 2$. In part (ii) of the theorem, we have assumed that $\Delta_{1}=\Delta_{3}=0$ (i.e. that the scalings are normalized). We also have assumed $\Delta_{0}=0$. Together with (2.9), this implies that $\Delta_{p}=0$ for all odd $p$, and

$$
\Delta_{p}=\operatorname{const}(-1)^{p / 2}(p+1)^{2} \sum_{l=1}^{p / 2} \frac{1}{2 l}
$$


for $p$ even. We have to show that this sequence leads to (1.5). With no loss of generality, we set the constant $=1$. Then,

$$
\begin{aligned}
\frac{\Delta(4 \operatorname{atn} z)}{1+z^{2}}=\sum_{p} \Delta_{p} z^{p} & =\frac{d}{d z} z \frac{d}{d z} z \sum_{p}\left((-1)^{p / 2}\left(\frac{1}{2}+\frac{1}{4}+\ldots+\frac{1}{p}\right)\right) z^{p} \\
& =-\frac{d}{d z} z \frac{d}{d z} z\left(\frac{1}{1+z^{2}}\left(\frac{1}{2} z^{2}-\frac{1}{4} z^{4}+-\ldots\right)\right) \\
& =-\frac{d}{d z} z \frac{d}{d z} z\left(\frac{1}{1+z^{2}} \frac{1}{2} \ln \left(1+z^{2}\right)\right) \\
& =\frac{2 z^{4}-4 z^{2}}{\left(1+z^{2}\right)^{3}}-\frac{\ln \left(1+z^{2}\right)}{2\left(1+z^{2}\right)^{3}}\left(1-6 z^{2}+z^{4}\right) .
\end{aligned}
$$

Letting $z=\tan (u / 4)$ proves (1.5) after some calculation.

This concludes the proof of part (ii) of the theorem; part (iii) is easy now:

\subsection{Completion of Proof}

By assumption, $\Delta(u, \varepsilon)$ does not contain a $u^{2}$ term. Up to scaling, we may again assume that $\Delta(u, \varepsilon)$ does not contain $u^{1}$ and $u^{3}$ terms either. Then, by (ii), $\Delta(u)=0$. So, one has $\mathscr{L} v=0$. At this point, the completeness of the eigenfunctions enters. (We have postponed all this, in order not to interrupt the discussion.) As a consequence of Theorem 12 below, the solution is a linear combination of $\partial_{t} u^{*}$ and $\partial_{x} u^{*}$. Of course, this is to be expected: translates of breathers in space and time are breathers again, the equation is not explicitly dependent on either $x$ or $t$. The important point is however that there are no further solutions to $\mathscr{L} v=0$. Our normalizations from the very beginning have killed the translations and imply $v=0\left[\right.$ because $\partial_{x} v\left(0, \frac{\pi}{2 \omega}\right)=0$ and $v(0,0)=0]$. Write (using analyticity in $\varepsilon$ )

$$
\varepsilon \Delta(u, \varepsilon)=\sum_{j} \varepsilon^{j} \Delta^{[j]}(u), \quad u(x, t, \varepsilon)=u^{*}(x, t ; m(\varepsilon))+\sum_{j} \varepsilon^{j} v^{[j]}(x, \tau) .
$$

We have already seen that $v=v^{[1]}=0$. The next condition $\left(O\left(\varepsilon^{2}\right)\right)$ is $\mathscr{L} v^{[2]}=$ $\Delta^{[2]}\left(u^{*}\right)$, i.e. the very same equation again. The argument can be repeated inductively.

This ends the proof of the theorem modulo the lemmas.

\section{Proof of the Lemmas}

\subsection{Proof of Lemma 6}

We make use of the following lemma on partial summation; it is standard, so no proof need be given.

\section{Lemma 9.}

$$
\sum_{q=-1}^{N} a_{q} b_{q}=-\sum_{q=0}^{N}\left(\left(a_{q}-a_{q-1}\right) \sum_{j=-1}^{q-1} b_{j}\right)+a_{N} \sum_{j=-1}^{N} b_{j} .
$$


Provided $a_{q} \sum_{-1}^{q} b_{j} \rightarrow 0$ as $q \rightarrow \infty$, therefore

$$
\sum_{q=-1}^{\infty} a_{q} b_{q}=-\sum_{q=0}^{\infty}\left(\left(a_{q}-a_{q-1}\right) \sum_{j=-1}^{q-1} b_{j}\right) .
$$

For better reference, we repeat (2.9):

$$
\Gamma_{p}:=(-1)^{\lfloor p / 2\rfloor} \frac{\Delta_{p}}{(p+1)^{2}}, \quad \mathrm{~B}_{p}:=p\left(\Gamma_{p}-\Gamma_{p-2}\right), \quad \mathrm{A}_{p}:=\mathrm{B}_{p+2}-\mathrm{B}_{p} .
$$

The sequence $\left(\Delta_{p}\right)$ was defined in (2.4), and from there, one has the estimate $\left|\Delta_{p}\right| \leq c(\varepsilon) /(\varrho-\varepsilon)^{p}$ for any small $\varepsilon>0$; the same estimates [with other $c(\varepsilon)$ ] hold for $\Gamma_{p}, \mathrm{~B}_{p}$, and $\mathrm{A}_{p}$. Write (2.6) in terms of $\Gamma_{p}$ :

$$
\begin{aligned}
\tilde{\mathscr{B}}_{n}\left(\frac{m}{\omega}\right)= & (-1)^{\lfloor n / 2\rfloor} \sum_{q=-1}^{\infty} \frac{\Gamma_{n+2 q}}{\left(m^{2}-1\right)^{q}} \\
& \times \prod_{r=0}^{q}\left[\frac{n^{2}-1}{4}+r(n+r) m^{2}\right] \frac{(n+2 q+1)^{2}}{(q+1) !(n+q+1) !} \\
& \times\left\{\frac{n^{2}+1}{2}(n+2 q+2)+n^{2}+\frac{(q+1)(n+q+1)}{\frac{n^{2}-1}{4}+q(n+q) m^{2}}\right. \\
& \left.\times\left(-(n+2 q-1)+\left(1-m^{2}\right)\left(\frac{n^{2}+1}{2}(n+2 q)-n^{2}\right)\right)\right\} .
\end{aligned}
$$

We identify $a_{q}$ with $\Gamma_{n+2 q}$ and let $b_{q}$ be the rest under the sum. At least for $n$ odd, we know that $\sum_{\jmath=-1}^{\infty} b_{\jmath}=0$, because $\Gamma_{p}=1$ ( $p$ odd) corresponds to the scaling solution $\Delta(u)=\sin u$. Indeed, one can immediately prove by induction on $q$ that:

$$
\begin{aligned}
\sum_{j=-1}^{q-1} b_{\jmath}= & \left(m^{2}-1\right)^{1-q} \prod_{r=0}^{q-1}\left[\frac{n^{2}-1}{4}+r(n+r) m^{2}\right] \\
& \times \frac{n+2 q}{q !(n+q) !}\left\{\frac{n^{2}-1}{2}\left((n+2 q)^{2}-1\right)\right\} .
\end{aligned}
$$

We skip the straightforward (though lengthy) calculation that is needed for this induction argument. For fixed $n$ and using that $\omega$ is bounded away from 0 by $\left|\frac{m}{\omega}\right|<\varrho$, we now see, by explicit estimate of the right-hand side, that

$$
\begin{aligned}
\left|\sum_{\jmath=-1}^{q-1} b_{j}\right| & \leq c_{1}(n)(1+|q|) \prod_{r=1}^{q-1}\left[\frac{c_{2}(n)}{r(n+r)}+\left|\frac{m^{2}}{\omega^{2}}\right|\right] \\
& \leq C(n, \varepsilon)\left(\left|\frac{m^{2}}{\omega^{2}}\right|+\varepsilon\right)^{q}
\end{aligned}
$$


so the boundary term $a_{q} \sum_{j=-1}^{q} b_{\jmath}$ is dominated by

$$
C^{\prime}(n, \varepsilon)\left(\left|\frac{m}{\omega}\right|^{2}+\varepsilon\right)^{q} /(\varrho-\varepsilon)^{n+2 q},
$$
and this tends to 0 for $\left|\frac{m}{\omega}\right|<\varrho$ and $\varepsilon$ sufficiently small. This justifies the partial
summation.

The previous induction, on which the partial summation is founded, is a purely algebraic argument, irrespective of whether $n$ is even or odd. The geometrically wellexplained scaling solution $\Gamma_{1}=\Gamma_{3}=\Gamma_{5}=\ldots=1$ has therefore an algebraic "shadow" $\Gamma_{0}=\Gamma_{2}=\Gamma_{4}=\ldots=1$, for which one also gets $\tilde{\mathscr{R}}_{n}^{\Delta} \equiv 0$. It is excluded from the theorem by the condition $\Delta_{0}=0$.

The formula for $\tilde{\mathscr{R}}_{n}$ has already significantly simplified:

$$
\begin{aligned}
\tilde{\mathscr{R}}_{n}\left(\frac{m}{\omega}\right)= & (-1)^{\lfloor n / 2\rfloor+1} \sum_{q=0}^{\infty} \frac{\mathrm{B}_{n+2 q}\left(m^{2}-1\right)^{1-q}}{q !(n+q) !} \prod_{r=0}^{q-1}\left[\frac{n^{2}-1}{4}+r(n+r) m^{2}\right] \\
& \times\left\{\frac{n^{2}-1}{2}\left((n+2 q)^{2}-1\right)\right\} .
\end{aligned}
$$

We now identify $a_{q}$ with $B_{n+2 q}$ and calculate $\sum b_{\jmath}$, again proving by induction that

$$
\begin{aligned}
& \sum_{\jmath=0}^{q-1} \frac{\left(m^{2}-1\right)^{1-\jmath}}{j !(n+j) !} \prod_{r=0}^{\jmath-1}\left[\frac{n^{2}-1}{4}+r(n+r) m^{2}\right]\left\{\frac{n^{2}-1}{2}\left((n+2 j)^{2}-1\right)\right\} \\
& \quad=2\left(n^{2}-1\right)\left(m^{2}-1\right)^{2-q} \prod_{r=0}^{q-1}\left[\frac{n^{2}-1}{4}+r(n+r) m^{2}\right] \frac{1}{(q-1) !(n+q-1) !} .
\end{aligned}
$$

With this, it follows

$$
\begin{aligned}
\tilde{\mathscr{R}}_{n}\left(\frac{m}{\omega}\right)= & (-1)^{\lfloor n / 2\rfloor} 2\left(n^{2}-1\right)\left(m^{2}-1\right) \sum_{q=0}^{\infty} \mathrm{A}_{n+2 q} \frac{\left(m^{2}-1\right)^{-q}}{q !(n+q) !} \\
& \times \prod_{r=0}^{q}\left[\frac{n^{2}-1}{4}+r(n+r) m^{2}\right]
\end{aligned}
$$

This completes the proof of the lemma.

The solution $\mathrm{B}_{3}=\mathrm{B}_{5}=\ldots=1$ contains the second scaling $\Delta(u)=u \cos u$. It also has an algebraic "shadow" $\mathrm{B}_{2}=\mathrm{B}_{4}=\ldots=1$. This shadow is responsible for (1.5).

Remark 7. Although we have insisted that $\Delta(0)=0$, it is interesting to calculate the first "shadow" $\Gamma_{0}=\Gamma_{2}=\Gamma_{4}=\ldots=1$. It corresponds to $\Delta(u)=\cos u$. This has a geometric meaning: under the shift $u \mapsto u-\varepsilon$, the sine Gordon equation goes into $u_{t t}-u_{x x}+\sin u=\varepsilon \cos u+O\left(\varepsilon^{2}\right)$.

Remark 8 . This and the following proof use extremely elementary techniques to prove rather messy formulas that come in like some deus ex machina. We do not know of a more intuitive proof that really explains these formulas, although the complete 
integrability of the sine Gordon equation is certainly behind them. The formulas that are proved by induction have first been looked for and guessed by playing around with Mathematica, a symbolic manipulation software [28].

\subsection{Proof of Lemma 7}

Note that the term under the product sign in (2.11) can be rewritten as $\tilde{q}(n+\tilde{q})-$ $r(n+r)=(\tilde{q}-r)(\tilde{q}+n+r)$. Every term in the sum (2.11) differs from the preceding one by a factor; therefore it is convenient to write the formula in a way similar to the Horner scheme. Here it is:

$$
\begin{aligned}
& R(n, \tilde{q})= \\
& \quad+\frac{1}{1(n+1)} \frac{-\left(n^{2}-1\right)}{(n+2 \tilde{q})^{2}-1}(\tilde{q}-1)(\tilde{q}+n+1) \cdot\left\{\mathrm{A}_{n+2}+\right. \\
& \quad+\frac{1}{2(n+2)} \frac{-\left(n^{2}-1\right)}{(n+2 \tilde{q})^{2}-1}(\tilde{q}-2)(\tilde{q}+n+2) \cdot\left\{\mathrm{A}_{n+4}+\right. \\
& +\frac{1}{(\tilde{q}-2)(n+\tilde{q}-2)} \frac{-\left(n^{2}-1\right)}{(n+2 \tilde{q})^{2}-1} 2(\tilde{q}+n+\tilde{q}-2) \cdot\left\{\mathrm{A}_{n+2(\tilde{q}-2)}+\right. \\
& \left.\left.\left.\left.+\frac{1}{(\tilde{q}-1)(n+\tilde{q}-1)} \frac{-\left(n^{2}-1\right)}{(n+2 \tilde{q})^{2}-1} 1(\tilde{q}+n+\tilde{q}-1) \cdot \mathrm{A}_{n+2(\tilde{q}-1)}\right\}\right\}\right\}\right\} .
\end{aligned}
$$

Obviously, $\mathrm{A}_{n+2 q}^{(Q)}$ as given in the lemma vanishes for $q=0,1, \ldots, Q-1$ and is different from 0 for $q=Q$. So the claim of the lemma is non-trivial only for $\tilde{q}>Q+1$, and the sum (2.11) really starts only at $q=Q$. The proof in this case is by induction along the Horner scheme (3.1). Start at the innermost level of parentheses with $X_{0}:=\mathrm{A}_{n+2(\tilde{q}+1)}$ and define for $l=1,2, \ldots, \tilde{q}-Q-1$ the result of the $l^{\text {th }}$ step in the scheme to be

$$
X_{l}:=X_{l-1} \frac{l(n+2 \tilde{q}-l)}{(\tilde{q}-l)(n+\tilde{q}-l)} \frac{-\left(n^{2}-1\right)}{(n+2 \tilde{q})^{2}-1}+\mathrm{A}_{n+2(\tilde{q}-l-1)}^{(Q)} .
$$

We now claim that

$$
\begin{aligned}
X_{l}= & \left(\frac{(n+2 Q+1)(n+2 Q+3)}{n^{2}-1}\right)^{\tilde{q}-l-1} \\
& \times \frac{(\tilde{q}-l-1) \ldots(\tilde{q}-Q) \cdot(\tilde{q}-Q-2) \ldots(\tilde{q}-Q-l-1)}{(\tilde{q}+n-l) \ldots(\tilde{q}+n+Q-l) \cdot(\tilde{q}+n+Q+1)} \\
& \times(n+2 \tilde{q}-1)(n+2 \tilde{q}+1),
\end{aligned}
$$

where the product $(\tilde{q}-Q-2) \ldots(\tilde{q}-Q-l-1)$ is to be interpreted as the empty product, 1 , for $l=0$. Using the formulas for $\mathrm{A}_{n+2 q}^{(Q)}$, it is straightforward to check 
this latter claim by induction on $l$ : For the induction step from $l-1$ to $l$, we have to show (after common factors have been cancelled) that

$$
\begin{aligned}
(\tilde{q}-Q-1)(n+2 \tilde{q}-2 l-1)(n+2 \tilde{q}-2 l+1)(n+\tilde{q}+Q+1) \\
\quad-(n+2 Q+1)(n+2 Q+3) l(n+2 \tilde{q}-l) \\
=(\tilde{q}+n+Q-l+1)(\tilde{q}-Q-l-1)(n+2 \tilde{q}-1)(n+2 \tilde{q}+1) .
\end{aligned}
$$

From the formula for $X_{l}$, it is obvious that $X_{\tilde{q}-Q-1}=0$, which proves the lemma.

\subsection{Proof of Lemma 8}

Suppose we can write $\alpha=\frac{N^{2}-1}{n^{2}-1}$ for integers $n$ and $N$. Then,

$$
\left(\sqrt{\alpha}-\frac{N}{n}\right)\left(\sqrt{\alpha}+\frac{N}{n}\right)=\frac{N^{2}-1}{n^{2}-1}-\frac{N^{2}}{n^{2}}=\frac{\alpha-1}{n^{2}} .
$$

As $n \rightarrow \infty, N / n \rightarrow \sqrt{\alpha}$, so we find

$$
\sqrt{\alpha}-\frac{N}{n} \sim \frac{A}{n^{2}} \quad \text { with } \quad A=\frac{\alpha-1}{2 \sqrt{\alpha}} .
$$

Unless $A=0$, i.e. $\alpha=1$, this implies that $\sqrt{\alpha}$ is irrational. On the other hand, take the difference of $n \sqrt{\alpha}-N \sim A / n$ and $(n+2) \sqrt{\alpha}-N^{\prime} \sim A /(n+2)$, and let $n \rightarrow \infty$. We find that $2 \sqrt{\alpha}$ is an integer, hence rational. So, $A$ must be 0 , proving the lemma.

\section{Ramifications}

\subsection{Analyticity Domain of $\tilde{\mathscr{R}}_{n}$}

In order to prove Theorem 2 , it is sufficient to show that $\tilde{\mathscr{R}}_{n}$ is analytic in as large a domain as $\Theta$ (and not only in as large a disc as $\Theta$, which we have already seen in Lemma 5). We first state this analyticity result and show why it implies Theorem 2, then we prove it.

We are going to consider two functions in the complex $z$-plane. The first is $\Theta$, which contains the information on the perturbation function $\Delta(\cdot)$ and is defined in (2.4). The second is $\mathscr{R}_{n}$. The connection between the arguments of $\mathscr{R}_{n}$ and $\Theta$ comes from the integral (4.1a) below: The argument of $\mathscr{R}_{n}$, namely $\frac{m}{\omega}$ enters into $\Theta\left(\frac{m \sin t}{\omega \operatorname{ch} x}\right)$ under the integral. The function $(x, t) \mapsto \frac{m \sin t}{\omega \operatorname{ch} x}$ has the critical values $\pm \frac{m}{\omega}$, which will play an important role in the subsequent discussion. This makes it quite natural to consider the functions $\mathscr{B}_{n}$ and $\Theta$ as functions in the same complex plane.

We prove the following

Theorem 10. Let $n \geq 2$ be fixed and $G$ be a simply connected domain which contains the origin and is point symmetric with respect to it. Let $\Theta(z)=\Delta(4 \operatorname{atn} z) /\left(1+z^{2}\right)=$ $\sum \Delta_{p} z^{p}$ be an even or odd function analytic in $G$ (the series being valid only 
in the largest disc $D \subset G$ of course). Then the function $\mathscr{R}_{n}\left(\frac{m}{\omega}\right)$ defined by (2.3) can be continued analytically into all of $G \backslash\{0\}$ with at most simple poles at $m / \omega=z(n, \tilde{q})= \pm i \sqrt{\left(n^{2}-1\right) /\left((n+2 \tilde{q})^{2}-1\right)},(\tilde{q}=1,2,3, \ldots)$. The possible pole at $z(n, \tilde{q})$ fails to appear, if and only if the recursion $R(n, \tilde{q})=0$ holds.

In particular, if $\mathscr{R}_{n}$ vanishes identically in $G$, then $R(n, \tilde{q})=0$ for all $\tilde{q}$ such that $z(n, \tilde{q}) \in G$. See (2.11) for the formula for $R(n, \tilde{q})$.

The parity assumption is no loss of generality, since $\mathscr{B}_{n}$ depends only on the odd part of $\Theta$, if $n$ is $\begin{gathered}\text { odd } \\ \text { even }\end{gathered}$ Neither is the assumption that $G$ should be simply connected: If it is multiply connected, we may apply the theorem to any simply connected subdomain. But then, $\mathscr{R}_{n}$ may be defined only on the universal cover of $G$, even if $\Theta$ is single-valued on $G$.

This theorem immediately implies Theorem 2 . The persistence assumptions of the latter force $\mathscr{B}_{n}$ to vanish identically for every $n$, and from this moment, the proof is the same as for Theorem 1 .

Proof of Theorem 10. First rewrite (2.3):

$$
\begin{aligned}
\mathscr{B}_{n}\left(\frac{m}{\omega}\right) & =\int_{-\infty}^{\infty} \int_{0}^{2 \pi / \omega} \chi_{n, \pm k_{n}}(x, t) \Delta\left(u^{*}(x, t)\right) d t d x \\
& =\frac{1}{m \omega} \int_{-\infty}^{\infty} \int_{0}^{2 \pi} \Theta\left(\frac{m \sin t}{\omega \operatorname{ch} x}\right) \Xi\left(x, t ; \frac{m}{\omega}\right) e^{i n t} e^{i \kappa(m / \omega) x} d x d t
\end{aligned}
$$

where

$$
\Xi_{n}\left(x, t ; \frac{m}{\omega}\right)=\frac{\kappa\left(\frac{m}{\omega}\right)}{2} \text { th } x-i \frac{\kappa^{2}\left(\frac{m}{\omega}\right)-1}{4}+\frac{n}{2} \frac{\sin t \cos t}{\operatorname{ch}^{2} x}-i \frac{n^{2}+1}{4} \frac{\sin ^{2} t}{\operatorname{ch}^{2} x}
$$

and

$$
\kappa(z)=\frac{\sqrt{n^{2}-1-z^{2}}}{z} \text { and } \Theta(z)=\frac{\Delta(4 \tan z)}{1+z^{2}} .
$$

In the second line of (4.1a), we have replaced the integration with respect to $t$ and $x$ by integration with respect to $\omega t$ and $m x$, and the new variables of integration are again called $x$ and $t$. In all the rest of this section, the variables $x$ and $t$ will denote these scaled space and time variables.

The term under the intgegral (4.1a) is a function of the three variables

$$
z=\frac{m}{\omega} \in G \backslash\{0\}, \quad t \in \mathbb{C} / 2 \pi \mathbb{Z}, \quad x \in \mathbb{C}^{\times}:=\mathbb{C} \backslash\{ \pm i \pi / 2, \pm 3 i \pi / 2, \pm 5 i \pi / 2, \ldots\}
$$

and is analytic in these three variables as long as the argument of $\Theta$ is contained in $G$. However, it is not globally uniquely valued, but has branch points at $\frac{m}{\omega}= \pm \sqrt{n^{2}-1}$ because of the term $\kappa(m / \omega)$.

We shall define $\mathscr{\circ}_{n}$ by integrating the same integrand as for $\mathscr{R}_{n}$ over a different domain of integration: the product of two closed loops, hence a compact manifold. 
This domain of integration can be deformed homotopically depending on $\frac{m}{\omega}$; that will do the analytic continuation. Afterwards, it will become clear that $\stackrel{\circ}{\mathscr{R}}_{n}$ is (except for a regular, nonvanishing factor) the same as $\tilde{\mathscr{B}}_{n}$.

Given $z_{0}$, define $G_{z_{0}}:=\left\{(t, x) \in \mathbb{C} / 2 \pi \mathbb{Z} \times \mathbb{C}^{\times} \mid z_{0} \frac{\sin t}{\operatorname{ch} x} \in G\right\}$. Moreover, we denote $G / z_{0}:=\left\{z / z_{0} \mid z \in G\right\}$ and similarly for $G$ multiplied with other numbers.

By a cycle $\mathscr{Z}$ in $G_{z_{0}}$, we understand (in a narrower sense than usual) a diffeomorphism from a 2-torus $S^{1} \times S^{1} \ni(\tau, \xi)$ into $G_{z_{0}}(t, x)$. It can be identified with a 2-cycle in the usual sense of homology theory by means of a triangulation of the 2-torus. With our narrower definition, homotopy of cycles is well-defined (and homotopic implies homologous).

We construct cycles for our purposes as follows: First we define $\mathscr{E}$ to be the product of a rectangular path $C=\partial([-\hat{x}, \hat{x}] \times i \cdot[0, \pi])$ in the complex $x$-plane, and $[0,2 \pi]$ considered as a loop $c$ in the $t$-cylinder $\mathbb{C} / 2 \pi \mathbb{Z}$. If $\left|z_{0}\right|$ is sufficiently small, this cycle will lie in $G_{z_{0}}$.

With this definition, $\{\sin t \mid t \in c\}=[-1,1]$, and $\{1 / \operatorname{ch} x \mid x \in C\} \subset[-1,1] \cup$ $B_{\delta}(0)$ for some small ball $B_{\delta}(0)$. Both of these sets are contained in $G / z_{0}$, provided $\left|z_{0}\right|$ is sufficiently small.

We deform $c$ a little, say into $c^{\prime}=[-i \varepsilon, 2 \pi-i \varepsilon]$ such that the curve $\tilde{\gamma}:=$ $\left\{\sin t \mid t \in c^{\prime}\right\}$ (which now goes around \pm 1 positively) still lies in $G / z_{0}$. (Actually, $\tilde{\gamma}$ is an ellipse.) We assume that the width of $C$ has been chosen large enough that the closure of $B_{\delta}(0)$ lies inside $\tilde{\gamma}$. Then, there is still some space for deforming $C$ into $C^{\prime}$ such that $\gamma:=\left\{1 / \operatorname{ch} x \mid x \in C^{\prime}\right\}$ still lies inside $\tilde{\gamma}$, but surrounds \pm 1 and 0 in negative orientation and is symmetric with respect to the origin. See Fig. 2. We define the cycle $\mathscr{Z}^{\prime}$ to be $c^{\prime} \times C^{\prime}$,
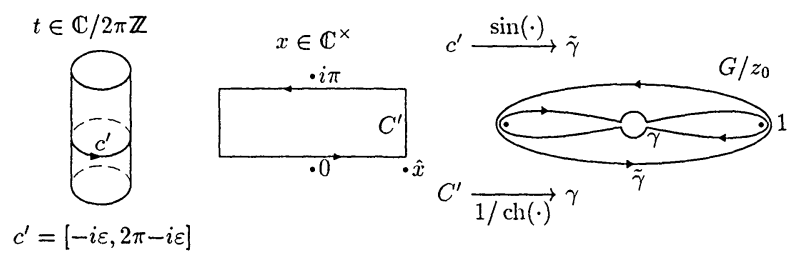

Fig. 2. The cycle $\mathbb{E}^{\prime}$

Generalizing $\mathscr{Z}^{\prime}$, we shall construct a cycle $\mathscr{Z}^{*}$ in $G_{z_{0}}$ for any $z_{0} \in G \backslash\{0\}$. The map $(z, t, x) \mapsto z \frac{\sin t}{\operatorname{ch} x}$ is continuous, $(t, x)$ varies over a compact set, and $G$ is open. Therefore, if $\mathbb{Z}^{*}$ is any cycle in $G_{z_{0}}$, it is also a cycle in $G_{z}$ for $z$ in some neighbourhood of $z_{0}$.

We now give the construction of $\mathscr{Z}^{*}$ : Let $s$ be a symmetric curve that connects -1 to $1 G / z_{0}$, and let $\gamma^{*}: S^{1} \rightarrow G / z_{0}, \xi \mapsto \gamma^{*}(\xi)$ surround $s$ with negative orientation. $\gamma^{*}$ is homotopic to $\gamma^{\prime}$ in $\mathbb{C} \backslash\{0, \pm 1\}$, and by lifting the homotopy $\gamma^{*} \sim \gamma^{\prime}$, we define

$$
C^{*}: S^{1} \rightarrow \mathbb{C}^{\times}, \quad \xi \mapsto \operatorname{arcosh} 1 / \gamma^{*}(\xi) .
$$

Next, we want to construct $\tilde{\gamma}^{*}$ homotopic to $\tilde{\gamma}$ and define $c^{*}$ from it. We do not care to preserve the property that $\gamma$ lies inside $\tilde{\gamma}$ for $\gamma^{*}$ and $\tilde{\gamma}^{*}$. We also allow that $\tilde{\gamma}^{*}=\tilde{\gamma}_{\xi}^{*}$ depends on $\xi$. 


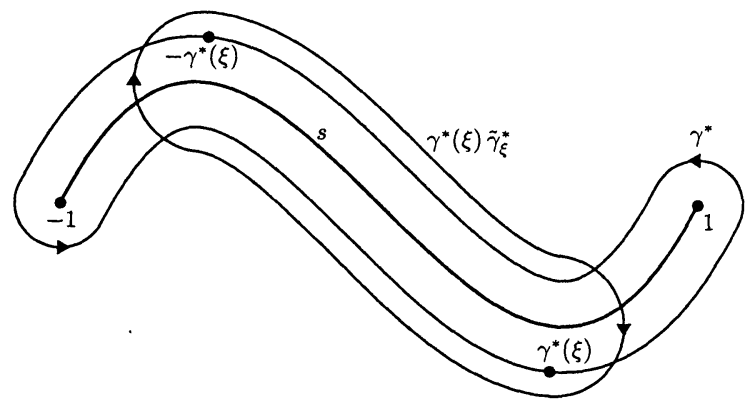

Fig. 3. The cycle $\mathscr{Z}^{*}$. All loops lie in a simply connected neighbourhood of the segment $s$ in $G / z_{0}$

To this end, for given $\xi$, we construct a loop in a tubular neighbourhood of $s$ that goes around $\pm \gamma^{*}(\xi)$ in positive direction, and we define this loop to be $\gamma^{*}(\xi)$ times the loop $\tilde{\gamma}_{\xi}^{*}$ (see Fig.3). This latter loop therefore goes around \pm 1 in positive direction and is homotopic to $\tilde{\gamma}$ in $\mathbb{C} \backslash\{ \pm 1\}$. So we define, by lifting the homotopy,

$$
c_{\xi}^{*}: S^{1} \rightarrow \mathbb{C} / 2 \pi \mathbb{Z}, \quad \tau \mapsto \arcsin \tilde{\gamma}_{\xi}^{*}(\tau),
$$

and from it the cycle

$$
\mathscr{Z}_{z_{0}}^{*}=\mathbb{Z}^{*}: S^{1} \times S^{1} \rightarrow \mathbb{C} / 2 \pi \mathbb{Z} \times \mathbb{C}^{\times}, \quad(\tau, \xi) \mapsto\left(c_{\xi}^{*}(\tau), C^{*}(\xi)\right) .
$$

This is a cycle in $G_{z_{0}}$, because $\sin c_{\xi}^{*}(\tau) / \operatorname{ch} C^{*}(\xi)=\tilde{\gamma}_{\xi}^{*}(\tau) \gamma^{*}(\xi) \in G / z_{0}$ by construction. Consequently, it is also a cycle in $G_{z}$, for $z$ sufficiently close to $z_{0}$; and it is homotopic to the cycle $\mathscr{Z}_{z}^{*}$ constructed in the same way for such a $z$ instead of $z_{0}$. For those $z_{0}$ for which $\mathscr{Z}^{\prime}$ was defined, $\mathscr{Z}_{z_{0}}^{*}$ is homotopic to $\mathscr{Z}^{\prime}$.

Having constructed the cycle $\mathscr{Z}^{*}=\mathscr{Z}_{z_{0}}^{*}$, we can define for $z$ in a neighbourhood of $z_{0}$ :

$$
\begin{gathered}
\stackrel{\circ}{\mathscr{B}}_{n}(z):=f_{n}(z) \frac{z}{1+z^{2}} e^{\pi \kappa(z) / 2} \iint_{\mathbb{Z}^{*}} \Theta\left(z \frac{\sin t}{\operatorname{ch} x}\right) \Xi(x, t ; z) e^{i n t} e^{i \kappa(z) x} d x d z, \\
\kappa(z)=\frac{\sqrt{n^{2}-1-z^{2}}}{z}, \quad f_{n}(z):=\left\{\begin{array}{ll}
\frac{2}{\sqrt{n^{2}-1-z^{2}}} & \text { for } n \text { even } \\
1 & \text { for } n \text { odd }
\end{array} .\right.
\end{gathered}
$$

These definitions coincide in the overlap of different neighbourhoods due to the homotopy properties established above. So, $\mathscr{\circ}_{n}$ is well-defined in $G \backslash\{0\}$.

Now, all terms under the integral are analytic functions of $z \in G$ [and the integrand has quadratic branch points $z= \pm \sqrt{n^{2}-1}$ due to the term $\left.\kappa(z)\right]$. Therefore, $\stackrel{\circ}{\mathscr{R}}_{n}(z)$ is an analytic function of $z$ on the universal cover of $G \backslash\left\{0, \pm \sqrt{n^{2}-1}\right\}$. As of now, it might e.g. have an essential singularity at 0 . The exponential in front of the integral is included in the definition to avoid this, as we shall see in a moment. For $z=\frac{m}{\omega}$, it holds $z /\left(1+z^{2}\right)=m \omega$. This factor and $f_{n}$ are also included for the purpose of regularization.

Our method of analytic continuation is appropriate for studying the global behaviour, but not for studying the behaviour near $z=0$. For this purpose, Lemma 5 is available. 
For $z$ small enough, we may take $\mathscr{Z}$ instead of $\mathscr{Z}^{*}$ and let the width of the rectangle $C$ in the $x$-plane tend to infinity. One finds that

$$
\stackrel{\circ}{\mathscr{B}}_{n}(z)=f_{n}(z)\left(\frac{z}{1+z^{2}}\right)^{2}\left(e^{\pi \kappa(z) / 2}-(-1)^{n} e^{-\pi \kappa(z) / 2}\right) \mathscr{R}_{n}(z) .
$$

Comparison of this formula with the splitting result of Lemma 5 shows after a short calculation that

$$
\stackrel{\circ}{\mathscr{R}}_{n}(z)=\pi^{2} i^{n-1} \prod_{r=-\left\lfloor\frac{n-1}{2}\right\rfloor}^{-1}\left[\frac{(n+2 r)^{2}-1}{4} z^{2}+\frac{n^{2}-1}{4}\right] \tilde{\mathscr{R}}_{n}(z) .
$$

In particular, $\stackrel{\circ}{\mathscr{R}}_{n}$ is regular near $z=0$.

From the fact that $\mathscr{R}_{n}$ does not depend on the sign of $\pm k_{n}$ [see (2.3)], it follows that $\mathscr{R}_{n}$ has no branch points at $m= \pm k_{n}$. Therefore, by (4.3), $\stackrel{\circ}{R}_{n}$ has no branch points at $z= \pm \sqrt{n^{2}-1}$ either. Hence, $\mathscr{R}_{n}$ is analytic in all of $G$, except for possible simple poles at $\pm i$ [introduced by the coefficient in (4.2)].

The analytic continuation property for $\mathscr{\mathscr { R }}_{n}$ implies the one for $\mathscr{R}_{n}$ immediately: The possible poles of $\mathscr{B}_{n}$ at $z(n, \tilde{q})$ arise when one solves (4.3) for $\mathscr{R}_{n}$. We have already seen that they are compensated by zeros of $\tilde{\mathscr{R}}_{n}$ (inside the disc of convergence of the series $\sum \Delta_{p} z^{p}$ ) if and only if the recursions are satisfied. On the other hand, simple poles of $\mathscr{\mathscr { B }}_{n}$ at $\pm i$ do not give rise to poles of $\mathscr{B}_{n}$; this follows again from (4.3). This proves the analyticity properties claimed for $\mathscr{B}_{n}$.

However, it is not completely trivial that the recursion $R(n, \tilde{q})=0$ is necessary and sufficient everywhere in $G$ for the possible pole of $\mathscr{B}_{n}$ at $z(n, \tilde{q})$ to vanish, because the recursions were originally defined through a series that will not converge everywhere in $G$. Here is the argument:

Consider $z^{*}=z(n, \tilde{q})$ and decompose $\Theta=\Theta_{1}+\Theta_{2}$, where $\Theta_{1}$ is the truncated Taylor series (hence trivially convergent everywhere) and $\Theta_{2}$ is the rest. The truncation is chosen to be at such high an order that all Taylor coefficients that enter into $R(n, \tilde{q})$ are already included in $\Theta_{1}$. Then $\mathscr{R}_{n}$ is regular at $z^{*}$, if and only if $0=\stackrel{\circ}{\mathscr{R}}_{n}^{\Theta}\left(z^{*}\right)=\stackrel{\circ}{\mathscr{B}}_{n}^{\Theta_{1}}\left(z^{*}\right)+\stackrel{\circ}{\mathscr{R}}_{n}^{\Theta_{2}}\left(z^{*}\right)$. Now, $\Theta_{2}(z)=O\left(z^{n+2 \tilde{q}+1}\right)$ as $z \rightarrow 0$. Therefore, the integral in (4.1a) converges whereever $\left|\operatorname{Im} \kappa\left(\frac{m}{\omega}\right)\right|<n+2 \tilde{q}+1$. For $\frac{m}{\omega}=z^{*}$, this condition is satisfied: $\left|\operatorname{Im} \kappa\left(z^{*}\right)\right|=n+2 \tilde{q}$. Therefore, $\mathscr{R}_{n}^{\Theta_{2}}$ is analytic near $z^{*}$, i.e. $\stackrel{\circ}{\mathscr{O}}_{n}^{\Theta_{2}}\left(z^{*}\right)=0$. So, $\mathscr{R}_{n}$ is regular at $z^{*}$, if and only if $\stackrel{\circ}{\mathscr{B}}_{n}^{\Theta_{1}}\left(z^{*}\right)=0$, hence

$$
0=\tilde{\mathscr{R}}_{n}^{\Theta_{1}}\left(z^{*}\right)=R^{\Theta_{1}}(n, \tilde{q})=R^{\Theta}(n, \tilde{q}) .
$$

This concludes the proof of Theorem 10 and hence of Theorem 2, too.

A different construction of the cycles has been used in [11]. The one given here is simpler. 


\subsection{More on the Recursion}

An instructive result on the recursions is hidden in Remark 3: if for fixed $n$, infinitely many of the recursions $R(n, \tilde{q})=0$ are satisfied, then all but finitely many of them are satisfied, because $\tilde{\mathscr{B}}_{n} \equiv 0$ in a neighbourhood of $z=0$. On the other hand, it is easy to construct recursively sequences that satisfy infinitely many recursions $R(n, \tilde{q})=0$, but at the same time fail to satisfy another infinitely many of them. The only way for this not to be a contradiction is that all those sequences are not sequences of Taylor coefficients of an analytic function, i.e. grow faster than $\alpha^{k}$ for any $\alpha$. This phenomenon has to arise regardless by how much the sequence fails the recursions, whereas by Lemma 7, sequences that satisfy all but finitely many recursion exactly grow only geometrically. This proves

Theorem 11. The recursive calculation of a sequence $\left(\mathrm{A}_{n+2 q}\right)$ from initial conditions and the recursions $R(n, \tilde{q})=0$ for $\tilde{q} \geq \tilde{q}_{\min }$ is ill-conditioned.

Next, there is a remarkable connection between the poles at $z(n, \tilde{q})$ encountered here and poles encountered by Kichenassamy [16]. He considers the global problem $u_{t t}-u_{x x}+g(u)=0$ and constructs formal breather solutions that are series expansions in $m^{k} \frac{\cos n \omega t}{\mathrm{ch}^{l} m x}$. In his divergence proof, he argues that if this series converges, then the coefficient functions $u_{l}(\omega t ; m)$ of a different series $\sum u_{l}(\omega t ; m) e^{-l m x}$, which normally have poles in the complex $m$-plane, cannot have these poles. (This second series represents a stable manfiold expansion.) These poles are exactly at the points $\frac{m}{\omega}=z(n, \tilde{q})$, too.

Their vanishing determines nonlinear recursions for the Taylor coefficients $g_{k}$ of $g(u)$. One can linearize these conditions about the coefficients of $g(u)=\sin u$. We have observed, but not proved, the following phenomena:

The linearization of Kichenassamy's condition from the $n^{\text {th }}$ Fourier component of $u_{l}$ is a multiple of $R(n, \tilde{q})$, where $n+2 \tilde{q}=l$. If "accidentally" $z(n, \tilde{q})=z\left(n^{\prime}, \tilde{q}^{\prime}\right)$ for some $n^{\prime}>n$, then Kichenassamy's condition at $\left(n^{\prime}, l^{\prime}\right)$ contains the one at $(n, l)$ as a factor, and its linearization about $g(u)=\sin u$ vanishes.

The true connection between both types of vanishing pole conditions is not yet understood. Understanding it should be a useful step towards obtaining global nonexistence results for breather families to nonlinear Klein Gordon equations. It would also be desirable to have a lemma for Kichenassamy's recursions that reduces to Lemma 7 in the linearization about $g(u)=\sin u$. If it exists, it will most probably be the key for strengthening his result that the formal series cannot converge in a certain domain $(|m|<1 / \sqrt{2}+\varrho)$. A strengthened theorem should exclude convergence even in a small domain $(|m|<\varrho$ for any $\varrho)$ by coping with the same difficulty encountered in the present work for small $\varrho$ and overcome by Lemma 7 .

\section{Appendix: On the Unperturbed Sine Gordon Equation}

In this appendix, we collect formulas for the unperturbed sine Gordon equation. They are obtained by means of soliton theory. The essential idea in this theory is to associate with the sine Gordon equation for $u$ a linear problem (interpreted as a scattering problem) in which $u$ plays the role of a potential. Spectral properties of a particular solution $u$ to the sine Gordon equation are encoded in the "scattering data" of the 
scattering problem with this potential $u$, similarly as the properties of a function are encoded in its Fourier transform, and $u$ can be retrieved from the scattering data $[1,19]$. References for the sine Gordon equation in particular are $[2,20,13,26]$.

The reader need not understand how the formulas are obtained; for our purposes, it is sufficient that these formulas, once given, can be checked by straightforward elementary calculations, which are however very lengthy and tedious. Limitations of space do not permit a detailed account of the techniques that produce the formulas. The reader who desires such an account can obtain a copy of the author's thesis [11], which contains a detailed one.

\subsection{Formulas}

We need bounded solutions to the linearized sine Gordon equation, and here they are: For every $\lambda$, one has functions $\Phi(x, t, \lambda)=\left[\begin{array}{l}\Phi_{1} \\ \Phi_{2}\end{array}\right]$ such that

$$
\partial_{t}\left(e^{-\imath \Omega(\lambda) t} \Phi\right)=\left[\begin{array}{cc}
0 & 1 \\
\partial_{x x}-\cos u & 0
\end{array}\right]\left(e^{-i \Omega(\lambda) t} \Phi\right),
$$

where

$$
\Omega=\Omega(\lambda)=2 \lambda+\frac{1}{8 \lambda}, \quad k=k(\lambda)=2 \lambda-\frac{1}{8 \lambda}, \quad \Omega(\lambda)^{2}-k(\lambda)^{2}=1 .
$$

They are given by the formulas

$$
\begin{aligned}
\Phi_{1}(x, t, \lambda) e^{-\imath \Omega(\lambda) t}= & \frac{\lambda}{n(\lambda)^{2}} e^{-\imath k(\lambda) x-\imath \Omega(\lambda) t}\left(1+\frac{m^{2} s^{2}}{\omega^{2} C^{2}}\right)^{-1} m^{2} \\
& \times\left\{-\frac{k(\lambda)}{2 m} \frac{S}{C}-\frac{\Omega(\lambda)}{2 \omega} \frac{c s}{C^{2}}-i\left[\left(\frac{k(\lambda)}{2 m}\right)^{2}-\frac{1}{4}\right]\right. \\
& \left.-i\left[\left(\frac{\Omega(\lambda)}{2 \omega}\right)^{2}+\frac{1}{4}\right] \frac{s^{2}}{C^{2}}\right\}, \\
\Phi_{2}(x, t, \lambda) e^{-\imath \Omega(\lambda) t}= & \partial_{t}\left(\Phi_{1}(x, t, \lambda) e^{-\imath \Omega(\lambda) t}\right),
\end{aligned}
$$

where

$$
C=\operatorname{ch} m x, \quad S=\operatorname{sh} m x, \quad c=\cos \omega t \quad s=\sin \omega t
$$

and

$$
n(\lambda)=\frac{\lambda}{2}(k(\lambda)+i m), \quad a(\lambda)=\frac{n(-\lambda)}{n(\lambda)}=\frac{k(\lambda)-i m}{k(\lambda)+i m} .
$$

The interpretation of $k(\lambda)$ and $\Omega(\lambda)$ as wave number and frequency is immediate; $a(\lambda)$ is the important entry in the $S$-matrix of the associated scattering problem; for informal purposes, it may be considered as the encoding of the breather $u^{*}$ in spectral data. $n(\lambda)$ is merely a comfortable abbreviation.

For real $\lambda, \Phi(\cdot, \cdot, \lambda)$ is bounded. $\Phi_{1}$ is asymptotic to the free Klein Gordon waves $-i / \lambda e^{-i k(\lambda) x-\imath \Omega(\lambda) t}$ as $x \rightarrow-\infty$ and $-i / \lambda a^{2}(\lambda) e^{-\imath k(\lambda) x-\imath \Omega(\lambda) t}$ as $x \rightarrow+\infty$. For non-real $\lambda, \Phi(\cdot, \cdot, \lambda)$ grows exponentially for either $x \rightarrow+\infty$ or $x \rightarrow-\infty$, with the important exception of those $\lambda$ for which $a(\lambda)$ vanishes, i.e.

$$
\lambda_{ \pm}=\frac{ \pm \omega+i m}{4}, \quad k\left(\lambda_{ \pm}\right)=i m, \quad \Omega\left(\lambda_{ \pm}\right)= \pm \omega, \quad a\left(\lambda_{ \pm}\right)=0 .
$$


These exceptional values are characteristic for the breather; they correspond to the bound states of the associated scattering problem. One has

$$
\Phi_{1}\left(x, t, \lambda_{ \pm}\right) e^{-i \Omega\left(\lambda_{ \pm}\right) t}=-2( \pm \omega-i m)\left(1+\frac{m^{2} s^{2}}{\omega^{2} C^{2}}\right)^{-1}\left\{e^{\mp i \omega t} \frac{i}{C} \mp \frac{s}{C^{2}} e^{m x}\right\}
$$

There are adjoint solutions $\Phi^{\mathrm{A}}$, which obey similar formulas:

$$
\partial_{t}\left(e^{i \Omega(\lambda) t} \Phi^{\mathrm{A}}\right)=\left[\begin{array}{cc}
0 & -\partial_{x x}+\cos u \\
-1 & 0
\end{array}\right]\left(e^{\imath \Omega(\lambda) t} \Phi^{\mathrm{A}}\right)
$$

for $\Phi_{1}^{\mathrm{A}}(x, t, \lambda)=\Phi_{2}(-x,-t, \lambda)$ and $\Phi_{2}^{\mathrm{A}}(x, t, \lambda)=\Phi_{1}(-x,-t, \lambda)$.

The (bi)-orthogonality and completeness relations for these functions are of basic importance. They are stated by McLaughlin and Scott in [20]:

For $t$ fixed (and suppressed in the notation), either of the following families of functions forms a basis of $L^{2}\left(\mathbb{R} \rightarrow \mathbb{R}^{2}\right)$ :

$$
\begin{aligned}
& \Phi(\cdot, \lambda) \quad \text { for } \lambda \text { real } \quad \Phi^{\mathrm{A}}(\cdot, \lambda), \\
& \left.\begin{array}{l}
\Phi\left(\cdot, \lambda_{k}\right) \\
\dot{\Phi}\left(\cdot, \lambda_{k}\right)
\end{array}\right\} \text { for } \lambda_{k} \in\left\{\lambda_{-}, \lambda_{+}\right\}\left\{\begin{array}{l}
\Phi^{\mathrm{A}}\left(\cdot, \lambda_{k}\right) \\
\dot{\Phi}^{\mathrm{A}}\left(\cdot, \lambda_{k}\right)
\end{array}\right. \text {. }
\end{aligned}
$$

$\left(\Phi^{\mathrm{A}}\right)$ is the adjoint basis to $(\Phi)$; more precisely:

$$
\begin{aligned}
& \left\langle\Phi^{\mathrm{A}}(\cdot, \mu) \mid \Phi(\cdot, \lambda)\right\rangle=\frac{4 \pi i}{\lambda} a^{2}(\lambda) \delta(\lambda-\mu), \\
& \left.\begin{array}{c}
\left\langle\Phi^{\mathrm{A}}\left(\cdot, \lambda_{j}\right) \mid \dot{\Phi}\left(\cdot, \lambda_{k}\right)\right\rangle \\
= \\
\left\langle\dot{\Phi}^{\mathrm{A}}\left(\cdot, \lambda_{\jmath}\right) \mid \Phi\left(\cdot, \lambda_{k}\right)\right\rangle
\end{array}\right\}=-\frac{2}{\lambda_{\jmath}} \dot{a}^{2}\left(\lambda_{j}\right) \delta_{j k}, \\
& \left\langle\dot{\Phi}^{\mathrm{A}}\left(\cdot, \lambda_{\jmath}\right) \mid \dot{\Phi}\left(\cdot, \lambda_{k}\right)\right\rangle=\left(-2 \frac{\dot{a}\left(\lambda_{j}\right) \ddot{a}\left(\lambda_{j}\right)}{\lambda_{j}}+2 \frac{\dot{a}^{2}\left(\lambda_{\jmath}\right)}{\lambda_{j}^{2}}\right) \delta_{\jmath k}, \\
& \text { all other }\langle\cdots \mid \cdots\rangle=0 \text {, }
\end{aligned}
$$

where $\lambda, \mu$ are real, and $\lambda_{k}, \lambda_{\jmath}$ are either $\lambda_{+}$or $\lambda_{-}$, the zeros of $a(\cdot)$. A minor misprint in Table 1 of [20] has been corrected in the second of the above formulas. We use Dirac's bra and ket notation; $\left\langle\Phi^{\mathrm{A}} \mid \Phi\right\rangle$ simply means $\int_{-\infty}^{\infty} \Phi^{\mathrm{A}^{\mathrm{T}}}(x) \Phi(x) d x$, and $|\Phi(\cdot, \lambda)\rangle\left\langle\Phi^{\mathrm{A}}(\cdot, \lambda)\right|$ is the projection operator onto $\Phi(\cdot, \lambda)$ (for real $\lambda$ the derivative of the spectral family with respect to $\lambda)$.

Moreover, the following completeness relation holds:

$$
\begin{gathered}
\frac{1}{4 \pi i} \int_{\Gamma}|\Phi(\cdot, \lambda)\rangle\left\langle\Phi^{\mathrm{A}}(\cdot, \lambda)\right| \frac{\lambda}{a^{2}(\lambda)} d \lambda=\text { Identity } \\
\frac{1}{4 \pi i} \int_{\Gamma} \Phi(x, \lambda) \Phi^{\mathrm{A}^{\mathrm{T}}}(y, \lambda) \frac{\lambda}{a^{2}(\lambda)} d \lambda=\left[\begin{array}{ll}
1 & 0 \\
0 & 1
\end{array}\right] \delta(x-y) .
\end{gathered}
$$

Here the integration over $\Gamma$ is a shorthand notation. $\Gamma$ is a path coming from real $-\infty$ and going to real $+\infty$, but passing through the upper $\lambda$ half plane above $\lambda_{ \pm}$. 
It can then be deformed into the real axis leaving contributions from the residues of the poles of $\lambda / a^{2}(\lambda)$. The functions $\Phi$ and $\Phi^{\mathrm{A}}$ are analytic in the upper half plane, thus no other contributions arise. Tacitly assuming this deformation to be performed, the integral really involves exactly those $\lambda$ that contribute to the claimed bases. In the case where $a$ has only simple zeros (as here $\lambda_{ \pm}$) in the upper half plane, the completeness relation reads therefore:

$$
\begin{aligned}
& \frac{1}{4 \pi i} \int_{-\infty}^{\infty} \Phi(x, \lambda) \Phi^{\mathrm{A}^{\mathrm{T}}}(y, \lambda) \frac{\lambda}{a^{2}(\lambda)} d \lambda \\
& \quad-\frac{1}{2} \sum_{j}\left\{\left(-\lambda_{j} \frac{\ddot{a}_{j}}{\dot{a}_{\jmath}^{3}}+\frac{1}{\dot{a}_{j}^{2}}\right) \Phi\left(x, \lambda_{j}\right) \Phi^{\mathrm{A}^{\mathrm{T}}}\left(y, \lambda_{\jmath}\right)\right. \\
& \left.\quad+\frac{\lambda_{j}}{\dot{a}_{j}^{2}}\left(\dot{\Phi}\left(x, \lambda_{\jmath}\right) \Phi^{\mathrm{A}^{\mathrm{T}}}\left(y, \lambda_{j}\right)+\Phi\left(x, \lambda_{j}\right) \dot{\Phi}^{\mathrm{A}^{\mathrm{T}}}\left(y, \lambda_{j}\right)\right)\right\} \\
& =\left[\begin{array}{ll}
1 & 0 \\
0 & 1
\end{array}\right] \delta(x-y) .
\end{aligned}
$$

The orthogonality relations are consequences of Wronskian identities for the solutions of the associated scattering problem, so their proof (for any $u$ ) is comparatively elementary, though lengthy. A sample of these calculations is worked out in [20]. Or else check them explicitly in the way described below for the completeness relations.

If one assumes that any function can be expanded in terms of the functions claimed to be a basis (i.e. completeness), then the coefficients follow from the orthogonality relations (as in ordinary Fourier series); in other words, the form of the completeness relation is also elementary, provided one has completeness. The hard problem is to prove completeness. The proof is not carried out in [20].

In fact, for the sine Gordon equation in characteristic coordinates, $u_{X T}=\sin u$, Kaup has given a completeness proof for the squared eigenfunctions together with their $\lambda$-derivatives at zeros of $a(\lambda)$ in [15]. (Our $\Phi$ are squared eigenfunctions.) Alas, it is not obvious how this result should imply completeness for the sine Gordon equation in laboratory coordinates. The squared eigenfunctions are equivalent in both cases, but the dual pairing is quite different; it involves integration over $x$ ( $t$ fixed) in one case, but integration over $X$ ( $T$ fixed) in the other. To the knowledge of the author, no formal proof for the completeness relation in laboratory coordinates has been written down in the literature in the case where $x$ varies over the real line, although no reasonable doubt has been cast on its validity either. Completeness in laboratory coordinates when $x$ varies over a finite interval with periodic boundary and parity conditions is implicitly proved in [4]. Their proof does not make use of the squared eigenfunction formalism, but uses the Hamiltonian structure of the equations: skew-orthogonality with respect to the symplectic structure and the families of $n$ dimensional invariant tori in the phase space.

We do not give a general proof for completeness either, but we have checked (5.9) by explicit calculation in the case of the breather. This is sufficient to ensure that our results are founded on full mathematical rigor. In fact, it suffices to check (5.9) for some fixed $t$, say $t=0$. It then automatically holds for all $t$ due to the time evolution equations (5.1) and (5.5). The next section gives an outline of the calculations involved. 


\subsection{Outline of a Direct Completeness Proof for the Breather Case}

Equation (5.9) is a matrix equation with four entries. With no loss of generality, we discuss the $1-1$ entry for $t=0$. Let us first consider the contribution coming from the integrals over real $\lambda$ : we have a factor

$$
\frac{\lambda^{3}}{16 n(\lambda)^{2} n(-\lambda)^{2}} e^{i k(\lambda)(y-x)}=\frac{1}{\lambda[k(\lambda)+i m]^{2}[k(\lambda)-i m]^{2}} e^{i k(\lambda)(y-x)} .
$$

Since it is $k(\lambda)$ that appears in the exponential, we want to introduce $k=k(\lambda)$ as the variable of integration. The mapping $\lambda \mapsto k(\lambda)$ is 2 -to-1, so we work with the variables $k$ and $\Omega=\Omega(\lambda)$ simultaneously, subject to the condition $\Omega^{2}=k^{2}+1$ [cf. (5.2)]. Then,

$$
\lambda=\frac{k+\Omega}{4}, \quad d \lambda=\frac{1}{4}\left(1+\frac{k}{\Omega}\right) d k, \quad \Omega^{2}=k^{2}+1, \quad \frac{\Omega(\lambda) d \lambda}{\lambda}=d k,
$$

$\int_{-\infty}^{\infty} \ldots d \lambda$ splits into two halves $\int_{-\infty}^{0} \ldots d \lambda+\int_{0}^{\infty} \ldots d \lambda$, each of which is transformed into $\int_{-\infty}^{\infty} \ldots d x$.

Multiplying out the terms in the braces in the formulas for $\Phi^{\mathrm{A}}(x, 0, \lambda)$, we get

$$
\begin{aligned}
& \frac{2}{4 \pi i} \int_{-\infty}^{\infty} \frac{e^{\imath k(y-x)} d k}{\left(k^{2}+m^{2}\right)^{2}}\left[i\left(k^{2}+m^{2}\right)^{2}-4 i k^{2} m^{2}\right. \\
& \quad+\left(k^{2}-m^{2}\right)\left(2 m k(\operatorname{th} m x-\operatorname{th} m y)+\frac{2 i m^{2}}{\operatorname{ch}^{2} m y}\right) \\
& \left.\quad+4 i m^{2} k^{2} \text { th } m x \text { th } m y+4 m^{3} k \frac{\operatorname{th} m x}{\operatorname{ch}^{2} m y}\right] .
\end{aligned}
$$

The first term in the above integral gives the desired $\delta(y-x)$ contribution because of the standard formula $\int_{-\infty}^{\infty} e^{i k(y-x)} d k=2 \pi \delta(y-x)$. The remaining integrals are evaluated separately for $y>x$ and $y<x$ by the calculus of residues. For example,

$$
\int_{-\infty}^{\infty} \frac{e^{i k(y-x)}}{\left(k^{2}+m^{2}\right)^{2}} d k=\frac{\pi}{2 m^{3}}(1+m|y-x|) \exp (-m|y-x|) .
$$

In the end, one gets the same result for both signs of $y-x$, namely that the regular contribution to $(5.10)$ is

$$
-\frac{m}{\operatorname{ch} m x \operatorname{ch} m y}+\frac{m^{2}(y-x) \text { th } m y}{\operatorname{ch} m x \operatorname{ch} m y} .
$$

Calculating the residual contributions in (5.9) is mere algebra, though quite lengthy. The symbolic manipulation software Mathematica has been used for this calculation. The term with $\Phi \Phi^{\mathrm{A}}$ gives

$$
\frac{2 m \text { th } m y}{\operatorname{ch} m x \operatorname{ch} m y\left(m^{2}-1\right)}
$$


whereas the term with $\dot{\Phi} \Phi^{\mathrm{A}}+\Phi \dot{\Phi}^{\mathrm{A}}$ gives

$$
\frac{2 m\left(1-m^{2}\right)(1+m \text { th } m y(x-y))-2 m \text { th } m y}{\operatorname{ch} m x \operatorname{ch} m y\left(m^{2}-1\right)} \text {. }
$$

Thus $-\frac{1}{2}$ times the sum of both indeed cancels the regular contributions from the integral, and the 1-1 matrix entry is as claimed. The other entries are obtained in the same way.

\subsection{General Solution of the First Order Equation}

In this section, we use the completeness relation for the functions $\Phi$ and $\Phi^{\mathrm{A}}$ to deduce that the necessary conditions for the solvability of $\mathscr{L} v=\Delta\left(u^{*}\right)$ that were given in Lemma 5 are also sufficient. This implies that we have done as good as one can with first order perturbation theory in the case of the exceptional perturbatrion (1.5): One really has persistence up to first order for it.

Furthermore, the discussion yields that the time and space derivatives of the sine Gordon breather are the only time periodic solutions to $\mathscr{L} \chi=0$ that decay as $x \rightarrow \pm \infty$. We have used this in Sect. 2.6.

Theorem 12. Let $u^{*}=4$ atn $\frac{m \sin \omega t}{\omega \operatorname{ch} m x}$ be the sine Gordon breather, let $\omega<\frac{1}{2}$ and $T:=2 \pi / \omega$, and consider the equation

$$
\mathscr{L} v=\left(\partial_{t}^{2}-\partial_{x}^{2}+\cos u^{*}\right) v=f(x, t)
$$

and assume that $f$ is T-periodic in $t$, decays exponentially as $x \rightarrow \pm \infty$ and is real analytic. This is to be solved for $v$ subject to the boundary conditions $v \rightarrow 0$ as $x \rightarrow \pm \infty$ and $v$ is T-periodic in $t$. Also assume that the compatibility conditions

$$
\int_{-\infty}^{\infty} \int_{0}^{T} f(x, t) \chi_{n, k_{n}}(x, t) d t d x=0
$$

are satisfied for all integers $n \neq 0$ with the functions $\chi_{n}$ given in Lemma 4. Then, (5.11) plus boundary conditions has a 2-dimensional space $v_{0}+\operatorname{ker} \mathscr{L}$ of solutions, where $\operatorname{ker} \mathscr{L}$ is spanned by the time-and space derivatives of $u^{*}$.

Remark. We have already noted earlier that for the interesting case, where $f(x, t)=$ $\Delta\left(u^{*}(x, t)\right)$, all compatibility conditions are satisfied if they are satisfied for $n \geq 2$.

We need the formulas (5.6)-(5.9). As before, it is a good strategy for understanding the formulas for $\Phi$ to look first at the exponentials and to consider everything else as Nature's camouflage.

Proof of the Theorem. Write the equation $\mathscr{L} v=f(x, t)$ in the form

$$
\partial_{t}\left[\begin{array}{c}
v \\
v_{t}
\end{array}\right]=\left[\begin{array}{cc}
0 & 1 \\
\partial_{x}^{2}-\cos u^{*} & 0
\end{array}\right]\left[\begin{array}{c}
v \\
v_{t}
\end{array}\right]+\left[\begin{array}{c}
0 \\
f(x, t)
\end{array}\right]
$$


For fixed $t$, any sufficiently ${ }^{2}$ regular and sufficiently decaying (as $x \rightarrow \pm \infty$ ) function $\left[\begin{array}{c}v \\ w\end{array}\right]$ can be expanded in squared eigenfunctions of the scattering problem for the sine Gordon equation. So we write

$$
\begin{aligned}
{\left[\begin{array}{c}
v \\
v_{t}
\end{array}\right](x, t)=} & \int_{-\infty}^{\infty} \hat{v}(\lambda, t) e^{-i \Omega(\lambda) t} \Phi(x, t, \lambda) d \lambda \\
& +\sum_{ \pm} \hat{v}_{ \pm}(t) e^{-i \Omega_{ \pm} t} \Phi_{ \pm}(x, t)+\sum_{ \pm} \hat{v}_{ \pm}(t) e^{-i \Omega_{ \pm} t} \dot{\Phi}_{ \pm}(x, t) .
\end{aligned}
$$

[ $\Phi_{ \pm}(x, t)$ stands for $\Phi\left(x, t, \lambda_{ \pm}\right)$, and a similar notation is used for other functions]. We abbreviate the first component of (5.13) as

$$
v(x, t)=v_{\text {cont }}(x, t)+v_{\text {disc }}(x, t)
$$

treating separately the contributions from the continuous and discrete spectrum. According to the formulas (5.7) and (5.8), it holds

$$
\begin{gathered}
\hat{v}(\lambda, t)=\frac{\lambda}{4 \pi i a^{2}(\lambda)} \int_{-\infty}^{\infty} e^{i \Omega(\lambda) t} \Phi^{\mathrm{A}^{\mathrm{T}}}(x, t, \lambda)\left[\begin{array}{c}
v \\
v_{t}
\end{array}\right](x, t) d x \\
\hat{\dot{v}}_{ \pm}(t)=-\frac{\lambda_{ \pm}}{2 \dot{a}_{ \pm}^{2}} \int_{-\infty}^{\infty} e^{i \Omega(\lambda) t} \Phi_{ \pm}^{\mathrm{A}^{\mathrm{T}}}(x, t)\left[\begin{array}{c}
v \\
v_{t}
\end{array}\right](x, t) d x \\
\frac{-2 \dot{a}_{ \pm}^{2}}{\lambda_{ \pm}} \hat{v}_{ \pm}(t)+\left(\frac{-2 \dot{a}_{ \pm} \ddot{a}_{ \pm}}{\lambda_{ \pm}}+\frac{2 \dot{a}_{ \pm}^{2}}{\lambda_{ \pm}^{2}}\right) \hat{\dot{v}}_{ \pm}(t) \\
=\int_{-\infty}^{\infty} e^{i \Omega(\lambda) t} \dot{\Phi}^{\mathrm{A}^{\mathrm{T}}}(x, t, \lambda)\left[\begin{array}{c}
v \\
v_{t}
\end{array}\right](x, t) d x .
\end{gathered}
$$

First translate the differential equation for $v$ into equations for the Fourier coefficients $\hat{v}$. From (5.14a) and the differential equations (5.12) and (5.1) for $v$ and $e^{i \Omega t} \Phi^{\mathrm{A}}$, we get that

$$
\partial_{t} \hat{v}(\lambda, t)=\frac{\lambda}{4 \pi i a^{2}(\lambda)} \int_{-\infty}^{\infty} e^{i \Omega(\lambda) t} \Phi_{2}^{\mathrm{A}}(x, t, \lambda) f(x, t) d x .
$$

On the other hand, we want $v(x, t)=v(x, t+2 \pi / \omega)$. Therefore, we must have $\hat{v}(x, t+2 \pi / \omega)=e^{2 \pi i \Omega(\lambda) / \omega} \hat{v}(x, t)$. These two conditions together imply

$$
\begin{aligned}
\hat{v}(\lambda, t)= & \left(e^{2 \pi i \Omega(\lambda) / \omega}-1\right)^{-1} \frac{\lambda}{4 \pi i a^{2}(\lambda)} \\
& \times \int_{t}^{t+2 \pi / \omega} \int_{-\infty}^{\infty} e^{i \Omega(\lambda) t} \Phi_{2}^{\mathrm{A}}(x, t, \lambda) f(x, t) d x d t .
\end{aligned}
$$

\footnotetext{
2 Sufficiently means the same as in ordinary Fourier transforms: if you want classical integrals $\left(L^{1}-\right.$ integrands), assume $v \in C^{2} \cap L^{1}$, or else make $L^{2}$ theory, or Schwartz-space theory, or tempered distributions. All other terms that enter into the formulas besides the exponentials are analytic in a strip and tend to 1 rapidly as $x \rightarrow \pm \infty$, so all desired results can immediately be reduced to the identical results for classical Fourier analysis
} 
Here, the necessary conditions re-appear. For values of $\lambda$, where $\Omega(\lambda) \in \omega \mathbb{Z}, \hat{v}$ will have a pole, unless a zero of the integral compensates it. But when $\hat{v}$ has a pole on the real axis, the integral (5.13) calculating $v$ is not defined, so no solution $v$ can be found. Or else, one may consider $\hat{v}$ as a tempered distribution (defined by its Cauchy principal value), then the integral (5.13) has a meaning, but produces a tempered distribution $v$ again. This $v$ will in fact be a function, but will not decay as $x \rightarrow \pm \infty$. The compatibility conditions guarantee that this dilemma does not appear and $v$ exists as a decaying function.

The integral on the right-hand side of (5.15) is real analytic for $\lambda \neq 0$, because the integrand is $e^{i k(\lambda) x}$ times a function that decays exponentially. The integral decays exponentially as $k \rightarrow \pm \infty$, because the integrand is analytic in $x$. The same holds for the derivative with respect to $\lambda$ of this integral. From the fact that the compatibility conditions hold, we conclude that all poles of $\left(e^{2 \pi \imath \Omega / \omega}-1\right)^{-1}$ are compensated and $\hat{v}$ is analytic, and that $\hat{v}$ decays exponentially (as $|k(\lambda)| \rightarrow \infty$ ).

We get an equation completely analogous to (5.15) for $\partial_{t} \hat{\dot{v}}_{ \pm}$, together with the periodicity condition $\hat{\dot{v}}_{ \pm}(t+2 \pi / \omega)=\hat{\dot{v}}_{ \pm}(t)$. This gives another compatibility condition (which is however automatically satisfied in the case $f(x, t)=\Delta\left(u^{*}(x, t)\right.$ ) we are really interested in, as we noted immediately before Lemma 5 . Therefore

$$
\hat{\dot{v}}_{ \pm}(t)=\frac{\lambda_{ \pm}}{2 \dot{a}_{ \pm}^{2}} \int^{t} \int_{-\infty}^{\infty} e^{i \Omega(\lambda) t} \Phi_{2}^{\mathrm{A}}\left(x, t, \lambda_{ \pm}\right) f(x, t) d x d t
$$

where the indefinite integral over $t$ introduces a constant of integration, $\dot{c}_{ \pm}$.

For $\hat{v}_{ \pm}(t)$, we get the differential equation

$$
\begin{aligned}
\partial_{t} \hat{v}_{ \pm}(t)= & -i \dot{\Omega}\left(\lambda_{ \pm}\right) \hat{\dot{v}}_{ \pm}(t)-\frac{\lambda_{+}}{2 \dot{a}_{ \pm}^{2}} \int_{-\infty}^{\infty} e^{i \Omega_{ \pm} t} \dot{\Phi}_{2 \pm}^{\mathrm{A}} f(x, t) d x \\
& +\left(\frac{\lambda_{ \pm} \ddot{a}_{ \pm}}{2 \dot{a}_{ \pm}^{3}}-\frac{1}{2 \dot{a}_{ \pm}^{2}}\right) \int_{-\infty}^{\infty} e^{\imath \Omega_{ \pm} t} \Phi_{2 \pm}^{\mathrm{A}} f(x, t) d x
\end{aligned}
$$

together with the periodicity condition $\hat{v}(t+2 \pi / \omega)=\hat{v}_{ \pm}(t)$. We can (and must) use the previous constant of integration $\dot{c}_{ \pm}$to ensure this periodicity condition.

New constants of integration, $c_{ \pm}$, arise this time, and they cannot be determined from the differential equation, because $\Phi\left(\lambda_{ \pm}\right)$are in $\operatorname{ker} \mathscr{L}$. (They span all of ker $\mathscr{L}$, because no more free constants have appeared in the calculation.)

This proves the theorem.

Acknowledgements. The author wants to express thanks to H. McKean for making available an early version of [5] and also for useful discussions by mail correspondence.

The present work contains the first main result of the author's PhD thesis [11], carried out at the ETH and advised by J. Moser. The author wants to thank him for valuable discussions, which stimulated the research and improved the presentation of the results.

\section{References}

1. Ablowitz, M.J., Kaup, D.J., Newell, A.C., Segur, H.: The inverse scattering transform - Fourier analysis for nonlinear problems. Studies in Appl. Math. Vol. LIII, No. 4, 249-315 (1974)

2. Ablowitz, M.J., Kaup, D.J., Newell, A.C., Segur, H.: Method for solving the sine-Gordon equation. Phys. Rev. Lett. 30, 1262-1264 (1973) 
3. Barone, A., Esposito, F., Magee, C.J., Scott, A.C.: Theory and applications of the sine-Gordon equation. Rivista del Nuovo Cimento 1, 227-267 (1971)

4. Bikbaev, P.F., Kuksin, S.B.: Periodic boundary value problem for the sine-Gordon equation, its small Hamiltonian perturbations, and KAM deformations of finite gap tori. To appear in Algebra i analiz (St. Petersburg Mathematical Journal) 3, 3 (1992)

5. Birnir, B., McKean, H., Weinstein, A.: Nonexistence of breathers. Private communication of draft, 1990, to appear in Comm. Pure Appl. Math.

6. Coron, J.-M.: Période minimale pour une corde vibrante de longueur infinie. Comptes Rendues Acad. Sci. Paris A 294, 127-129 (1982); erratum A 295, 371 (1982)

7. Craig, W., Wayne, C.E.: Newton's method and periodic solutions of nonlinear wave equations. Preprint 1991

8. Craig, W., Wayne, C.E.: Nonlinear waves and the KAM theorem: nonlinear degeneracies. Preprint 1991

9. Delshams, A., Martinez-Seara, M.T.: An asymptotic expression for the splitting of separatrices of the rapidly forced pendulum. Preprint 1991

10. Denzler, J.: Nonpersistence of Breather solutions under perturbation of the sine Gordon equation. In: Proceedings on dynamical systems, Euler International Mathematical Institute, Sankt Petersburg, November 1991, ed. V.F. Lazutkin, to appear in Birkhäuser, Basel

11. Denzler, J.: Nonpersistence of Breathers for the perturbed sine Gordon equation. $\mathrm{PhD}$ thesis number 9954, ETH Zürich, Switzerland, 1992. Copy available from the author

12. Eleonskii, V.M., Kulagin, N.E., Novozhilova, N.S., Silin, V.P.: Asymptotic expansions and qualitative analysis of finite-dimensional models in nonlinear field theory. Teor. i Mat. Fizika 60, 3, 395-403 (1984)

13. Forest, M.G., McLaughlin, D.W.: Spectral theory for the periodic sine Gordon equation: a concrete viewpoint. J. Math. Phys. 23, 1248-1277 (1982)

14. Holmes, P., Marsden, J., Scheurle, J.: Exponentially small splittings of separatrices with applications to KAM theory and degenerate bifurcations. Contemp. Math. 81, 213-244 (1988)

15. Kaup, D.J.: Closure of the squared Zakharov-Shabat eigenstates. J. Math. Anal. and Appl. 54, 849-864 (1976)

16. Kichenassamy, S.: Breather solutions of the nonlinear wave equation. Comm. Pure Appl. Math. 44, 789-818 (1991)

17. Kuksin, S.B.: Quasiperiodic solutions of nearly integrable infinite-dimensional Hamiltonian systems. To appear in Springer Lect. Notes in Math.

18. Lazutkin, V.F., Schachmannski, I.G., Tabanov, M.B.: Splitting of separatrices for standard and semistandard mappings. Physica D 40, 235-248 (1989)

19. McLaughlin, D.W.: Four examples of the inverse method as a canonical transformation. J. Math. Phys. 16, 96-99 (1975)

20. McLaughlin, D.W., Scott, A.C.: Perturbation analysis of fluxon dynamics. Phys. Rev. A 18, 4, 1652-1680 (1978)

21. Marsden, J.E., McCracken, M.: The Hopf bifurcation and its applications. Berlin, Heidelberg, New York: Springer 1976

22. Scheurle, J.: Splitting of separatrices and chaos. Lect. in Appl. Math. 26, 561-571 (1990)

23. Segur, H., Kruskal, M.D.: Nonexistence of small amplitude Breather solutions in $\phi^{4}$ theory. Phys. Rev. Lett. 58, 747-750 (1987)

24. Siegel, C.L., Moser, J.K.: Lectures on celestial mechanics. Springer Grundlehren 187, 1971

25. Sigal, I.M.: Non-linear wave and Schrödinger equations. I. Instability of periodic and quasiperiodic solutions. Preprint 1991

26. Takhtadzhyan, L.A., Faddeev, L.D.: Essentially nonlinear one-dimensional model of classical field theory. Teor. i Mat. Fizika 21, 2, 160-174 (1974)

27. Weinstein, A.: Periodic nonlinear waves on a half-line. Commun. Math. Phys. 99, 385-388 (1985); erratum 107, 177 (1986)

28. Wolfram, S.: Mathematica; a system for doing mathematics on a Computer. Addison-Wesley 